\title{
Local GMM Estimation of Time Series Models with Conditional Moment Restrictions*
}

\author{
Nikolay Gospodinov ${ }^{\dagger}$ \\ Concordia University and CIREQ \\ Taisuke Otsu ${ }^{\ddagger}$ \\ Yale University
}

First Version: September 2008

Revised: December 2009

\begin{abstract}
This paper investigates statistical properties of the local generalized method of moments (LGMM) estimator for some time series models defined by conditional moment restrictions. First, we consider Markov processes with possible conditional heteroskedasticity of unknown forms and establish the consistency, asymptotic normality, and semi-parametric efficiency of the LGMM estimator. Second, we undertake a higher-order asymptotic expansion and demonstrate that the LGMM estimator possesses some appealing bias reduction properties for positively autocorrelated processes. Our analysis of the asymptotic expansion of the LGMM estimator reveals an interesting contrast with the OLS estimator that helps to shed light on the nature of the bias correction performed by the LGMM estimator. The practical importance of these findings is evaluated in terms of a bond and option pricing exercise based on a diffusion model for spot interest rate.
\end{abstract}

Keywords: Conditional moment restriction; Local GMM; Higher-order expansion; Conditional heteroskedasticity.

Jel Classification: C13; C22; G12.

\footnotetext{
${ }^{*}$ We would like to thank the editors, a co-editor, three anonymous referees, conference participants at the 2007 CIREQ conference on Generalized Method of Moments, the 2007 meeting of the Canadian Econometrics Study Group and the 2009 Joint Statistical Meetings for useful comments and suggestions. Financial support from FQRSC and SSHRC (Gospodinov) and National Science Foundation under SES-0720961 (Otsu) is gratefully acknowledged.

${ }^{\dagger}$ Corresponding author: Department of Economics, Concordia University, 1455 de Maisonneuve Blvd. West, Montreal, Quebec, H3G 1M8 Canada, email: nikolay.gospodinov@concordia.ca.

${ }^{\ddagger}$ Cowles Foundation and Department of Economics, Yale University, P.O. Box 208281, New Haven, CT, 06520-8281 USA, email: taisuke.otsu@yale.edu.
} 


\section{Introduction}

While modern economic theory typically implies a set of conditional moment restrictions, estimation of the model parameters is often performed using a framework based on unconditional moment restrictions such as the standard generalized method of moments (GMM). Despite its computational attractiveness, this GMM-based approach may result in efficiency losses and inconsistency that arises from possible nonidentifiability of the parameters of interest by the unconditional moment restrictions even when the conditional moment restrictions identify the parameters (Dominguez and Lobato, 2004). Time series regression models, for example, are usually defined in terms of a sequence of disturbances whose expectation conditional on a few recent lags of the data is assumed to be zero. This specification allows for conditional heteroskedasticity which is a stylized feature of many economic and financial time series data such as interest rates, exchange rates, asset returns, etc.

This paper studies estimation of conditional moment restriction models in a time series context. In particular, we focus on the local (conditional or smoothed) GMM (LGMM, hereafter) estimator that belongs to the class of localized versions of the generalized empirical likelihood estimator (Newey and Smith, 2004) introduced and developed by Kitamura, Tripathi and Ahn (2004), Smith (2007), and Antoine, Bonnal and Renault (2007) (see, e.g., Newey, 1990, 1993; Carrasco and Florens, 2000; and Donald, Imbens and Newey, 2003, for some alternative methods to estimate conditional moment restriction models). While all these papers investigate the properties of the local estimators for iid data, we study the first- and higher-order asymptotic properties of the LGMM estimator for strictly stationary and geometrically ergodic Markov processes.

First, we study the first-order asymptotic behavior of the LGMM estimator and show that the LGMM estimator is consistent and asymptotically normal, and attains the semi-parametric efficiency bound for conditional moment restrictions with a martingale difference structure derived in Carrasco and Florens (2004). We should note that semi-parametrically efficient moment-based estimators have been proposed by Carrasco, Chernov, Florens and Ghysels (2007), Kuersteiner (2001, 2002) and West, Wong and Anatolyev (2009) for more general time series processes. Although our Markov setup is more restrictive than the setups of these papers, our emphasis is on the bias property of the LGMM estimator in small samples and the Markov setup helps us to simplify the higher-order analysis of the estimator. Indeed, our simulation results for AR models with conditional heteroskedasticity suggest that the LGMM estimator is characterized by a smaller bias and mean squared error than these alternative estimators and, in some cases, even the infeasible GLS estimator.

Next, in order to explain the bias reduction property of the LGMM estimator, we consider 
explicitly the AR(1) model with iid errors and undertake a higher-order expansion for the LGMM estimator. In particular, while the bias of the OLS estimator is given by a single negative term of order $O\left(T^{-1}\right)$, the higher-order expansion of the LGMM estimator reveals the presence of two $O\left(T^{-1}\right)$ bias terms. We compare these terms with those of the OLS estimator and provide an approximation formula for one of them. Our numerical results show that for positively autocorrelated processes the two leading bias terms in the LGMM estimator tend to have opposite signs and similar magnitudes that offset each other. Also, this bias reduction is achieved without inflating the variance of the LGMM estimator and then the LGMM can potentially dominate the GLS and other efficient estimators in terms of mean squared errors. The economic significance of the bias reduction property of the LGMM estimator is assessed for derivative interest rate products whose prices exhibit strong sensitivity to the parameter that governs the persistence of the process. Interestingly, the LGMM estimator seems to outperform in terms of mean square error the infeasible optimal GMM as the LGMM tends to remove a substantial portion of the bias that arises from the highly persistent dynamics of the interest rate data.

Our paper complements the existing econometric and statistical literature which is concerned with extending the local estimators for moment condition models to dependent data. For example, Kitamura (1997) considers unconditional moment restrictions with weakly dependent data and proposes a non-local empirical likelihood estimator by using blocked (or local average) moment restrictions. Our paper has three important differences with Kitamura (1997): (i) while Kitamura (1997) focuses on unconditional moments, we consider conditional moment restrictions that imply an infinite number of unconditional moments, (ii) while Kitamura (1997) requires local averaging for moments to account for the long-run variance of the unconditional sample moments, we employ local averaging to nonparametrically approximate the conditional moment restrictions, and (iii) while Kitamura (1997) allows for general weakly dependent data, our analysis is restricted to Markov processes.

In a different but related strand of literature, Chen, Härdle and Li (2003) propose a goodness-offit test statistic based on the local empirical likelihood function to check the validity of conditional moment restrictions. Furthermore, Su and White (2003) adopt the local empirical likelihood framework to test conditional independence restrictions which imply sequences of conditional moment restrictions. Both papers focus on hypothesis testing with possibly dependent data and derive firstorder asymptotic properties of the local empirical likelihood-based test statistics. In contrast, we focus on point estimation of parameters in conditional moment restriction models and derive some first- and higher-order properties of the LGMM estimator. Finally, Gagliardini, Gourieroux and Renault (2007) extend the local estimation approach to estimate conditional moments of interest (derivative prices in their example) with dependent data when the conditional moment restrictions 
(say, $E\left[u_{1}\left(y_{t+1}, \theta_{0}\right) \mid x_{t}\right]=0$ ) and local moment restrictions (say, $E\left[u_{2}\left(y_{t+1}, \theta_{0}\right) \mid x_{t}=\bar{x}\right]=0$ for some given $\bar{x}$ ) coexist. While Gagliardini, Gourieroux and Renault (2007) use the LGMM approach to evaluate the local moments $E\left[u_{2}\left(y_{t+1}, \theta_{0}\right) \mid x_{t}=\bar{x}\right]=0$, they adopt the optimal instrumental variable approach of Chamberlain (1987) and Newey $(1990,1993)$ to accommodate the conditional moments $E\left[u_{1}\left(y_{t+1}, \theta_{0}\right) \mid x_{t}\right]=0$. Our setup differs from Gagliardini, Gourieroux and Renault (2007) since we do not consider the local moments $E\left[u_{2}\left(y_{t+1}, \theta_{0}\right) \mid x_{t}=\bar{x}\right]=0$ but utilize the LGMM framework to estimate the parameters $\theta_{0}$ in the conditional moments $E\left[u_{1}\left(y_{t+1}, \theta_{0}\right) \mid x_{t}\right]=0$. Also, the general theoretical arguments in Gagliardini, Gourieroux and Renault (2007) are mostly concerned with deriving the efficiency bounds in their setup. On the other hand, we are interested in establishing the first- and higher-order asymptotic properties of the LGMM estimator.

The rest of the paper is organized as follows. The next section describes the model and estimation procedure. Section 3 develops the first-order asymptotic theory for the LGMM estimator and reports some numerical properties of the estimator in finite samples. Section 4 derives the higher-order asymptotic expansion of the LGMM estimator in an AR(1) model with iid errors and analyzes the bias properties of the estimator. The bias terms of the LGMM and OLS estimators are evaluated by simulation. Section 5 assesses the economic significance of the bias reduction and the efficiency of the LGMM in the context of a bond and derivative pricing exercise. Section 6 concludes. All proofs are contained in the appendix.

\section{Model and Estimation Procedure}

Suppose that the univariate process of interest $\left\{r_{t}\right\}_{t=-\infty}^{\infty}$ on $\mathbb{R}$ is strictly stationary and geometrically ergodic ${ }^{1}$ and denote the conditional moment restrictions imposed by some economic theory as

$$
E\left[u\left(r_{t+1}, r_{t}, \ldots, r_{t-p+1}, \theta_{0}\right) \mid r_{t}, \ldots, r_{t-p+1}\right]=0,
$$

for each $t \in \mathbb{Z}=\{\ldots,-1,0,1, \ldots\}$, where $u: \mathbb{R}^{p+1} \times \Theta \rightarrow \mathbb{R}^{l}$ is a known function up to a vector of unknown parameters $\theta_{0} \in \Theta \subseteq \mathbb{R}^{k}$. Technical conditions that restrict the dependence structure of the process and ensure the validity of the estimation procedure are discussed in the next section.

One popular example of this framework is the AR(1) model with martingale difference errors

$$
r_{t+1}=\gamma_{0}+\gamma_{1} r_{t}+u_{t+1}, \quad E\left[u_{t+1} \mid r_{t}\right]=0,
$$

\footnotetext{
${ }^{1}$ Let $\left\{X_{t}\right\}_{t=-\infty}^{\infty}$ be a time-homogeneous Markov process with state space $\left(\mathbb{R}^{p}, \mathcal{B}\left(\mathbb{R}^{p}\right)\right)$, where $\mathcal{B}\left(\mathbb{R}^{p}\right)$ is a Borel $\sigma$-algebra of $\mathbb{R}^{p}$, and $m$-step transition probability $P^{m}(x, A)=P\left(X_{m} \in A \mid X_{0}=x\right)$ for $x \in A$ and $A \in \mathcal{B}\left(\mathbb{R}^{p}\right)$. The process $\left\{X_{t}\right\}_{t=-\infty}^{\infty}$ is geometrically ergodic if there exists a probability measure $\pi$ on $\left(\mathbb{R}^{p}, \mathcal{B}\left(\mathbb{R}^{p}\right)\right)$, a constant $0<\rho<1$, and a $\pi$-integrable non-negative measurable function $C(\cdot)$ such that $\left\|P^{m}(x, \cdot)-\pi(\cdot)\right\|_{\tau} \leq \rho^{m} C(x)$ for all $m \in \mathbb{N}$ and $x \in \mathbb{R}^{p}$, where $\|\cdot\|_{\tau}$ denotes the total variation norm (Carrasco and Chen, 2002; Meyn and Tweedie, $1993)$.
} 
for each $t \in \mathbb{Z}$. In this case, the moment function is specified as $u\left(r_{t+1}, r_{t}, \theta_{0}\right)=r_{t+1}-\gamma_{0}-\gamma_{1} r_{t}$ with $\theta_{0}=\left(\gamma_{0}, \gamma_{1}\right)$. For $\gamma_{1}>0$, this model can be regarded as a discrete-time specification of the diffusion process

$$
d r_{t}=\kappa\left(\mu-r_{t}\right) d t+\sigma\left(r_{t}\right) d W_{t}
$$

for each $t \in[0, \infty)$, where $\left\{W_{t}\right\}_{t \geq 0}$ is the standard Brownian motion, $\kappa\left(\mu-r_{t}\right)$ is the drift component, and $\sigma(\cdot)$ is the diffusion function. In this linear parametrization of the drift function, $\mu$ is the long-run unconditional mean of the process and $\kappa$ is the speed of mean reversion. The diffusion process (3) is often used to model the dynamics of spot interest rate whose parameters are inferred from the estimates of the discrete-time model (2) by setting $\gamma_{0}=\mu\left(1-e^{-\kappa}\right)$ and $\gamma_{1}=\left(1-e^{-\kappa}\right)$. In Section 5, we employ this model for a bond and derivative pricing exercise.

In order to simplify the notation, let $x_{t}=\left(r_{t}, \ldots, r_{t-p+1}\right)^{\prime}$ and $y_{t+1}=\left(r_{t+1}, x_{t}^{\prime}\right)^{\prime} .^{2}$ The conditional moment restriction model (1) is typically estimated by the GMM estimator based on the unconditional moment restrictions $E\left[g\left(y_{t+1}, \theta_{0}\right)\right]=E\left[\mathcal{A}\left(x_{t}, \theta_{0}\right) u\left(y_{t+1}, \theta_{0}\right)\right]=0$ with a matrix of instruments $\mathcal{A}\left(x_{t}, \theta_{0}\right)$, which is implied from the original model (1). For example, the (continuously updated) GMM estimator (Hansen, Heaton and Yaron, 1996) is defined as

$$
\hat{\theta}_{G M M}=\arg \min _{\theta \in \Theta}\left(\frac{1}{T-p} \sum_{t=p}^{T-1} g\left(y_{t+1}, \theta\right)\right)^{\prime} W_{T}(\theta)^{-1}\left(\frac{1}{T-p} \sum_{t=p}^{T-1} g\left(y_{t+1}, \theta\right)\right),
$$

where $W_{T}(\theta)=\frac{1}{T-p} \sum_{t=p}^{T-1} g\left(y_{t+1}, \theta\right) g\left(y_{t+1}, \theta\right)^{\prime}$ is an optimal weight matrix to estimate the parameters from the unconditional moment restrictions $E\left[g\left(y_{t+1}, \theta_{0}\right)\right]=0$.

In this paper, we pursue an alternative approach and use a localized version of the GMM estimator that operates directly on the conditional moment restriction (1). Let $w_{t j}=\mathbb{K}\left(\frac{x_{j}-x_{t}}{h}\right) / \sum_{i=p}^{T-1} \mathbb{K}\left(\frac{x_{i}-x_{t}}{h}\right)$ denote kernel weights, where $\mathbb{K}: \mathbb{R}^{p} \rightarrow \mathbb{R}$ is a kernel function and $h$ is a bandwidth parameter. Let $\mathbb{I}_{t T}=I\left\{\left|x_{t}\right| \leq c_{T}\right\}$ be a trimming term to deal with some bias problems of kernel estimators, where $I\{\cdot\}$ is the indicator function and $c_{T}$ is a sequence satisfying $c_{T} \propto T^{\xi}$ for some $\xi>0 .{ }^{3}$ The kernel estimator for the conditional moment $E\left[u\left(y_{t+1}, \theta\right) \mid x_{t}\right]$ is defined as $u_{T}\left(x_{t}, \theta\right)=\sum_{j=p}^{T-1} w_{t j} u\left(y_{j+1}, \theta\right)$ and the LGMM estimator minimizes its quadratic form, i.e.,

$$
\hat{\theta}_{L G M M}=\arg \min _{\theta \in \Theta} \sum_{t=p}^{T-1} \mathbb{I}_{t T} u_{T}\left(x_{t}, \theta\right)^{\prime} V_{T}\left(x_{t}, \theta\right)^{-1} u_{T}\left(x_{t}, \theta\right),
$$

where $V_{T}\left(x_{t}, \theta\right)=\sum_{j=p}^{T-1} w_{t j} u\left(y_{j+1}, \theta\right) u\left(y_{j+1}, \theta\right)^{\prime}$ is an optimal weight matrix to estimate the parameters from the conditional moment restrictions.

\footnotetext{
${ }^{2}$ If we have exogenous regressors $z_{t}$, the vectors $x_{t}$ and $y_{t}$ can be defined as $x_{t}=\left(r_{t}, \ldots, r_{t-p+1}, z_{t}^{\prime}\right)^{\prime}$ and $y_{t+1}=$ $\left(r_{t+1}, x_{t}^{\prime}, z_{t}^{\prime}\right)^{\prime}$.

${ }^{3}$ Alternatively, similar to Kitamura, Tripathi and Ahn (2004), the trimming term may be defined as $I\left\{\frac{1}{T-p} \sum_{j=p}^{T-1} \mathbb{K}\left(\frac{x_{j}-x_{t}}{h}\right) \geq c_{T}^{\prime}\right\}$ with some $c_{T}^{\prime} \rightarrow 0$, which trims observations that have small kernel density estimates. Although we employ the trimming term $\mathbb{I}_{t T}$ to simplify our technical argument, a similar but more lengthy argument for the alternative trimming term defined above will yield analogous results to ours.
} 
Smith (2007) shows that the LGMM estimator (5) belongs to the class of local Cressie-Read minimum distance estimators which also includes the local (conditional or smoothed) empirical likelihood estimator proposed by Kitamura, Tripathi and Ahn (2004). See Smith (2007) and Antoine, Bonnal and Renault (2007) for a detailed discussion and interpretation of these local estimators. ${ }^{4}$ In this study, we adopt the LGMM estimator (5) instead of the local empirical likelihood estimator due to technical (for higher-order analysis) and computational reasons (see, Antoine, Bonnal and Renault, 2007). However, our preliminary numerical experiments with the local empirical likelihood reveal only negligible differences for those estimators.

\section{First-Order Asymptotic Theory}

\subsection{Asymptotic Properties of LGMM Estimator}

In this section, we establish the consistency, asymptotic normality, and semi-parametric efficiency of the LGMM estimator for stationary, possibly nonlinear, and conditionally heteroskedastic $p$-th order Markov processes.

Assumption A1. The process $\left\{r_{t}\right\}_{t=-\infty}^{\infty}$ is a strictly stationary, absolutely regular, $p$-th order Markov process in $\mathbb{R}$ (i.e., $P\left(r_{t+1} \mid r_{t}, r_{t-1}, \ldots\right)=P\left(r_{t+1} \mid r_{t}, \ldots, r_{t-p+1}\right)$ for each $t \in \mathbb{Z}$ ) with mixing coefficients of order $O\left(\rho^{m}\right)$ for $m \in \mathbb{N}$ and some $0<\rho<1$.

Assumption A1 requires that the process $\left\{r_{t}\right\}_{t=-\infty}^{\infty}$ is strictly stationary and absolutely regular ( $\beta$-mixing) with exponentially decaying mixing coefficients. This assumption is used to invoke the central limit theorem for $U$-statistics with weakly dependent data (Fan and Li, 1999). Also, recall that if the process is absolutely regular, it is also strong $(\alpha-)$ mixing. For example, Masry and Tjøstheim (1995) establish the conditions for geometric $\alpha$-mixing of the conditionally heteroskedastic first-order Markov process

$$
r_{t+1}=\phi\left(r_{t}\right)+u_{t+1}, \quad u_{t+1}=\sigma\left(r_{t}\right) \varepsilon_{t+1},
$$

for each $t \in \mathbb{Z}$, where $\phi: \mathbb{R} \rightarrow \mathbb{R}$ denotes the conditional mean function $E\left[r_{t+1} \mid r_{t}\right], \sigma^{2}: \mathbb{R} \rightarrow(0, \infty)$ denotes the conditional variance function $\operatorname{Var}\left(r_{t+1} \mid r_{t}\right)$, and $\varepsilon_{t+1}$ is iid with $E\left[\varepsilon_{t+1}\right]=0$ and $E\left[\varepsilon_{t+1}^{2}\right]=1$ for $t \in \mathbb{Z}$. The model in (6) allows for various types of conditional heteroskedasticity but it should be stressed that the LGMM estimator does not require any knowledge of the explicit form of the skedastic function $\sigma^{2}(\cdot)$ to estimate the parameters in the conditional mean function $\phi(\cdot)$.

Furthermore, the process generated by (6) is geometrically ergodic if $\lim \sup _{|r| \rightarrow \infty} \frac{\phi^{2}(r)+\sigma^{2}(r)}{r^{2}}<1$ (Maercker, 1995; Meyn and Tweedie, 1993). If $E\left[r_{t+1} \mid r_{t}\right]=\theta_{0} r_{t}$ and $\sigma^{2}\left(r_{t}\right)=\omega_{0}+\beta_{0} r_{t}^{2}$, then

\footnotetext{
${ }^{4}$ Other papers that adopt a similar type of local weighting include Kuersteiner (2006), Lavergne and Patilea (2008), and Lewbel (2007).
} 
this condition becomes $\theta_{0}^{2}+\beta_{0}<1$; if $E\left[r_{t+1} \mid r_{t}\right]=\theta_{0} r_{t}$ and $\sigma^{2}\left(r_{t}\right)=\sigma_{0}^{2} r_{t}^{\gamma_{0}}$, then this condition becomes $\left|\theta_{0}\right|<1$ for $\gamma_{0} \in[0,2)$ and $\theta_{0}^{2}+\sigma_{0}^{2}<1$ for $\gamma_{0}=2$. For the case of $E\left[r_{t+1} \mid r_{t}\right]=\theta_{0} r_{t}$ and $\sigma^{2}\left(r_{t}\right)=\omega_{0}+\beta_{0} r_{t}^{2}$, Borkovec (2001) and Borkovec and Klüppelberg (2001) prove the geometric ergodicity and strict stationarity of $\left\{r_{t}\right\}_{t=-\infty}^{\infty}$ under the weaker conditions of symmetry of $\varepsilon_{t+1}$ and $E\left[\log \left|\theta_{0}+\varepsilon_{t+1} \sqrt{\beta_{0}}\right|\right]$ which allows for $\left|\theta_{0}\right| \geq 1$ and $E\left[u_{t+1}^{2}\right]=\infty$. Moreover, Ling (2004) establishes the asymptotic normality of the quasi maximum likelihood estimator of $\theta_{0}$ that holds even for $\left|\theta_{0}\right| \geq 1$ and $E\left[u_{t+1}^{2}\right]=\infty$. Carrasco and Chen (2002) provide some sufficient conditions for $\beta$-mixing of various GARCH and stochastic volatility models. Finally, Guégan and Diebolt (1994) discuss some challenges in verifying the probabilistic properties of higher-order Markov processes.

Let $|A|=\sqrt{\operatorname{trace}\left(A^{\prime} A\right)}$ be the Euclidean norm for a scalar, vector, or matrix $A$, and min eig $(A)$ and maxeig $(A)$ be the minimum and maximum eigenvalues for a matrix $A$, respectively. Let $f(\cdot)$ be the density function of $X_{t}, V(x, \theta)=E\left[u\left(y_{t+1}, \theta\right) u\left(y_{t+1}, \theta\right)^{\prime} \mid x_{t}=x\right]$, and $D(x, \theta)=$ $E\left[\partial u\left(y_{t+1}, \theta\right) / \partial \theta^{\prime} \mid x_{t}=x\right]$. The following definition is useful to summarize the boundedness and continuity conditions for the moment function $u$ and its derivatives.

Definition ( $\mathcal{D}$-bounded function). A function $a: \mathbb{R}^{p+1} \times A \rightarrow \mathbb{R}^{q}$ is called $\mathcal{D}$-bounded on $A$ with order $s$ if

(i) $a(y, \theta)$ is almost surely differentiable at each $\theta \in A$,

(ii) $\sup _{\theta \in A} E\left[\left|a\left(y_{t+1}, \theta\right)\right|^{s}\right]<\infty$ and $\sup _{\theta \in A} E\left[\left|\partial a\left(y_{t+1}, \theta\right) / \partial \theta^{\prime}\right|^{s}\right]<\infty$ for each $t=p, \ldots, T-1$,

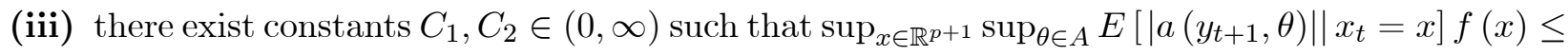
$C_{1}$ and $\sup _{x \in \mathbb{R}^{p+1}} \sup _{\theta \in A} E\left[\mid \partial a\left(y_{t+1}, \theta\right) / \partial \theta^{\prime} \| x_{t}=x\right] f(x) \leq C_{2}$ for each $t=p, \ldots, T-1$.

$\mathcal{D}$-boundedness assumes boundedness of the conditional and unconditional (higher-order) moments of the function $a$ and its derivative. These boundedness requirements guarantee sufficient regularity in order to apply a general uniform convergence theorem of Kristensen (2009); see Lemma A in Appendix A. Based on the above definition, Assumption A2 states the regularity conditions on the moment function $u$.

Assumption A2. Assume that

(i) $\Theta \subset \mathbb{R}^{k}$ is compact and $\theta_{0} \in \operatorname{int}(\Theta)$.

(ii) $E\left[u\left(y_{t+1}, \theta_{0}\right) \mid x_{t}\right]=0$ almost surely for each $t=p, \ldots, T-1$, and for each $\theta \in \Theta \backslash\left\{\theta_{0}\right\}$ and $t=$ $p, \ldots, T-1$, there exists a set $\mathbb{X}_{\theta} \subseteq \mathbb{R}^{p}$ such that $\operatorname{Pr}\left\{x_{t} \in \mathbb{X}_{\theta}\right\}>0$ and $E\left[u\left(y_{t+1}, \theta\right) \mid x_{t}=x\right] \neq$ 0 for all $x \in \mathbb{X}_{\theta}$. 
(iii) $E\left|x_{t}\right|^{1+s_{0}}<\infty$ for some $s_{0}>0 . u(y, \theta)$ and $u(y, \theta) u(y, \theta)^{\prime}$ are $\mathcal{D}$-bounded on $\Theta$ with order $s_{1}, s_{2}>2$, respectively. $\inf _{x \in \mathbb{R}^{p}} \inf _{\theta \in \Theta} \min \operatorname{eig}(V(x, \theta))>0$ and $\sup _{x \in \mathbb{R}^{p}} \sup _{\theta \in \Theta} \max \operatorname{eig}(V(x, \theta))<$ $\infty$. For each $\theta \in \Theta$ and $t=p, \ldots, T-1$, the derivatives of $f(x), V(x, \theta) f(x)$, and $E\left[u\left(y_{t+1}, \theta\right) \mid x_{t}=x\right] f(x)$ with respect to $x$ are uniformly continuous and bounded on $\mathbb{R}^{p}$.

(iv) There exists a neighborhood $\mathcal{N}$ around $\theta_{0}$ such that $\frac{\partial u}{\partial \theta_{k_{1}}}$ and $\frac{\partial^{2} u}{\partial \theta_{k_{1}} \partial \theta_{k_{2}}}$ are $\mathcal{D}$-bounded on $\mathcal{N}$ with order $s_{3}, s_{4}>2$ for each $k_{1}, k_{2}=1, \ldots, k$, respectively, and the derivatives of $E\left[\frac{\partial u\left(y_{t+1}, \theta\right)}{\partial \theta_{k_{1}}} \mid x_{t}=x\right] f(x)$ and $E\left[\frac{\partial^{2} u\left(y_{t+1}, \theta\right)}{\partial \theta_{k_{1}} \partial \theta_{k_{2}}} \mid x_{t}=x\right] f(x)$ with respect to $x$ are uniformly continuous and bounded on $\mathbb{R}^{p}$ for each $\theta \in \mathcal{N}, t=p, \ldots, T-1$, and $k_{1}, k_{2}=1, \ldots, k$. For some $\omega \in \mathbb{N}$, the $\omega$-th order derivatives ${ }^{5}$ of (each element of) $f(x), V\left(x, \theta_{0}\right) f(x)$, and $D\left(x, \theta_{0}\right) f(x)$ with respect to $x$ are uniformly continuous and bounded on $\mathbb{R}^{p}$. For each $t=p, \ldots, T-1$, the derivative of $E\left[\frac{\partial u\left(y_{t+1}, \theta_{0}\right)}{\partial \theta^{\prime}} u\left(y_{t+1}, \theta_{0}\right)^{\prime} \mid x_{t}=x\right] f(x)$ with respect to $x$ is uniformly continuous and bounded on $\mathbb{R}^{p}$.

Assumption A2 (i) is standard. Assumption A2 (ii) provides the identification condition for the true parameter $\theta_{0}$. Assumption A2 (iii) lists regularity conditions for the consistency of the LGMM estimator. Assumption A2 (iv) contains additional conditions to derive the asymptotic normality.

Let $a^{\mu}=\prod_{j=1}^{p} a_{j}^{\mu_{j}}$ for $p$-vectors $a$ and $\mu$ of real values and nonnegative integers, respectively. Define $\delta_{T}=\inf _{|x| \leq c_{T}} f(x)$. The conditions for the kernel function and bandwidth are summarized in the following assumption.

Assumption A3. Assume that

(i) $\mathbb{K}$ is uniformly bounded and satisfies $\int \mathbb{K}(a) d a=1, \int a^{\mu} \mathbb{K}(a) d a=0$ for each $\mu$ with $1 \leq$ $\sum_{j=1}^{p} \mu_{j} \leq \omega-1$, and $\int\left|a^{\mu} \mathbb{K}(a)\right| d a<\infty$ for each $\mu$ with $\sum_{j=1}^{p} \mu_{j}=\omega$. Also, there exist $C_{K}, L_{K} \in(0, \infty)$ such that either (a) $\mathbb{K}(a)=0$ for all $|a|>L_{K}$ and $\left|\mathbb{K}(a)-\mathbb{K}\left(a^{\prime}\right)\right| \leq$ $C_{K}\left|a-a^{\prime}\right|$ for all $a$ and $a^{\prime}$, or (b) $\mathbb{K}(a)$ has a uniformly bounded derivative and $|\partial \mathbb{K}(a) / \partial a| \leq$ $C_{K}|a|^{-v_{K}}$ for all $|a| \geq L_{K}$ and some $v_{K}>1$.

(ii) $h=h_{T}$ satisfies $\log T /\left(T h^{p}\right) \rightarrow 0, \log T /\left(\delta_{T}^{2} T^{1 / 2} h^{p}\right) \rightarrow 0, h^{p} / \delta_{T} \rightarrow 0$, and $T^{1 / 4} h^{\omega} / \delta_{T} \rightarrow 0$ as $T \rightarrow \infty$. Also, $c_{T} \propto T^{\xi}$ for some $0<\xi<\infty$.

Assumption A3 (i) imposes restrictions on the shape of the kernel function and is based on Kristensen (2009, Assumption A.6.1). To obtain reasonably fast convergence rates for bias components in kernel estimators, we use a higher-order ( $\omega$-th order) kernel. Combined with the condition

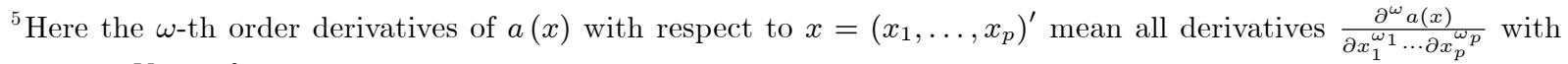
$\omega_{1}, \ldots \omega_{p} \in \mathbb{N}$ satisfying $\omega_{1}+\cdots \omega_{p}=\omega$.
} 
$T^{1 / 4} h^{\omega} / \delta_{T} \rightarrow 0$ in Assumption A3 (ii), the bias components of the kernel estimators are typically of order $o\left(T^{-1 / 4}\right)$. Assumption A3 (ii) contains conditions for the bandwidth $h$ and trimming constant $c_{T}$, which also controls $\delta_{T}$. The first condition $\log T /\left(T h^{p}\right) \rightarrow 0$ comes from Kristensen (2009, Theorem 1 ) by setting $\theta=1$ in his notation, which follows from exponential decay of the mixing coefficients in Assumption A1. The second condition $\log T /\left(\delta_{T}^{2} T^{1 / 2} h^{p}\right) \rightarrow 0$ is required to control the variance components for kernel estimators. The third and fourth conditions are used to control the bias components of kernel estimators. The last condition is on the trimming constant $c_{T}$. This condition is not as weak as it seems for two reasons. First, the weak requirement on $\xi$ is due to the exponentially decaying mixing coefficients in Assumption A1. If we allow polynomial decay in the mixing coefficients, we will have some upper bound for $\xi$. Second, since the rate of $c_{T}$ determines the rate of $\delta_{T}$, we cannot choose arbitrary the rate for $c_{T}$.

The first-order asymptotic properties of the LGMM estimator are established in the following theorem.

Theorem 1.

(a) Under Assumptions A1, A2 (i)-(iii), and A3,

$$
\hat{\theta}_{L G M M} \stackrel{p}{\rightarrow} \theta_{0}
$$

(b) Under Assumptions A1-A3,

$$
\begin{aligned}
& \qquad \sqrt{T}\left(\hat{\theta}_{L G M M}-\theta_{0}\right) \stackrel{d}{\rightarrow} N\left(0, \mathcal{I}\left(\theta_{0}\right)^{-1}\right), \\
& \text { where } \mathcal{I}\left(\theta_{0}\right)=E\left[D\left(x_{t}, \theta_{0}\right)^{\prime} V\left(x_{t}, \theta_{0}\right)^{-1} D\left(x_{t}, \theta_{0}\right)\right]
\end{aligned}
$$

Theorem 1 demonstrates the consistency and asymptotic normality of the LGMM estimator. Also, the form of the asymptotic variance $\mathcal{I}\left(\theta_{0}\right)^{-1}$ implies that the LGMM estimator attains the semi-parametric efficiency bound for $p$-th order Markov models derived in Carrasco and Florens (2004). The basic strategy of the proof of Theorem 1 is a modest modification of the arguments in Kitamura, Tripathi and Ahn (2004) and Antoine, Bonnal and Renault (2007) to our time series context using a uniform convergence theorem of Kristensen (2009) and central limit theorem for $U$-statistics of Fan and Li (1999). Although the result in this theorem is new in the literature, the main focus of the paper is to formally investigate the bias reduction property of the LGMM estimator in a time series model using higher-order analysis.

An alternative approach that achieves semi-parametric efficiency is based on the unconditional GMM estimator (4) with the optimal instruments $\mathcal{A}\left(x_{t}, \theta_{0}\right)=D\left(x_{t}, \theta_{0}\right)^{\prime} V\left(x_{t}, \theta_{0}\right)^{-1}$ (Chamberlain, 1987, and Newey, 1993, for iid data; Carrasco and Florens, 2004, for dependent data). As mentioned 
in the introduction, Carrasco, Chernov, Florens and Ghysels (2007), Kuersteiner (2001, 2002) and West, Wong and Anatolyev (2009) have developed efficient estimators in more general setups than ours. Our Markov framework, however, delivers analytical tractability of the estimator that allows us to study the finite-sample behavior of the estimator by higher-order expansions. Also, while the implementation of some efficient estimators could be quite involved, the LGMM estimator is characterized by some appealing properties from a practical point of view such as computational simplicity and speed.

\subsection{Finite-Sample Performance of LGMM Estimator}

To get some initial idea about the numerical properties of the LGMM estimator, we generate data from the $\operatorname{AR}(1)$ model

$$
r_{t+1}=\theta_{0} r_{t}+u_{t+1}, \quad u_{t+1}=\sigma_{t+1} \varepsilon_{t+1},
$$

for each $t=0,1, \ldots, T$, where $\varepsilon_{t+1} \sim i i d N(0,1)$ and $r_{0}$ is initialized from its stationary distribution. The skedastic function $\sigma_{t+1}$ is parametrized as an $\operatorname{ARCH}(1)$ process $\sigma_{t+1}=\sqrt{\left(1-\beta_{0}\right)+\beta_{0} u_{t}^{2}}$ with $\beta_{0}=0,0.5$, and $0.9 .^{6}$ The AR parameter $\theta_{0}$ is set equal to $0.4,0.7$, and 0.95 .

Although the true value of the intercept is zero, we do not impose this restriction in the estimation and the estimated model includes an intercept. The number of Monte Carlo replications is 10,000. In the practical implementation of the LGMM estimation, we standardize the conditioning variable and use the Gaussian kernel, which satisfies Assumption A3 (i). For the bandwidth parameter, we employ the rule of thumb choice of $h=1.06 T^{-1 / 5}$ but our preliminary simulation results suggest that the estimator is relatively insensitive to a wide range of bandwidth values. The LGMM estimation is performed without any trimming, i.e. $\mathbb{I}_{t T}=1$ for all $t .^{7}$

In addition to the LGMM estimator, we consider the OLS estimator and the infeasible GLS estimator which uses the true skedastic function as GLS weights. Furthermore, we compare the LGMM estimator to two other efficient estimators: optimal instrumental variables estimator for heteroskedastic AR models (Kuersteiner, 2002; West, Wong and Anatolyev, 2009) and Carrasco and Florens' (2000) efficient estimator for moment condition models. For the estimator of Carrasco and Florens (2000) (see also, Carrasco, Chernov, Florens and Ghysels, 2007, for the extension of the estimator to dependent data), we use a smoothing parameter ( $\alpha$ in their notation) of 0.02 and a standard normal integrating density. ${ }^{8,9}$

\footnotetext{
${ }^{6}$ In an earlier version of the paper, we also considered different forms of conditional heteroskedasticity. The results are qualitatively very similar and are available from the authors upon request.

${ }^{7}$ Our preliminary experiments showed that the effect of trimming in the simulation setups considered in the paper is negligible.

${ }^{8}$ We would like to thank Marine Carrasco for generously providing the codes for implementing the Carrasco-Florens estimator.

${ }^{9}$ The OLS estimator can be regarded as the simplest example of a non-local GMM estimator based on the un-
} 
The mean bias, median bias, standard deviation, and root mean squared error (RMSE) for all estimators are reported in Tables 1 and 2 for sample sizes $T=100$ and 500, respectively. As expected, the OLS estimator is characterized by a substantial downward bias that seems to increase with the degree of conditional heteroskedasticity. The optimal instrumental variable estimator exhibits similar behavior and in most cases its bias even exceeds the OLS bias which is consistent with the simulation results reported in Kuersteiner (2002). While the other three estimators are also biased, the magnitude of their bias is smaller and the bias tends to decrease as the conditional heteroskedasticity becomes stronger. Not surprisingly, the infeasible GLS estimator delivers the smallest standard errors among all estimators. Also, the GLS estimator provides a nontrivial bias correction and in several cases its bias is less than half of the OLS bias.

Compared to the OLS and optimal instrumental variables estimators, the Carrasco-Florens estimator also produces a bias reduction which is most effective when the persistence of the process is small and the conditional heteroskedasticity is strong. In terms of efficiency and RMSE, the Carrasco-Florens estimator substantially dominates the optimal instrumental variables estimator. Interestingly, the LGMM estimator is characterized by the smallest bias across all parametrization. The bias reduction for the LGMM estimator is particularly pronounced as the degree of conditional heteroskedasticity increases and in the $\mathrm{ARCH}$ case with $\beta_{0}=0.9$ (the last panels in Tables 1 and 2 ), this estimator is almost median unbiased. Furthermore, the LGMM estimator enjoys some significant efficiency gains and dominates in several cases the infeasible GLS in terms of RMSE.

One interesting finding that emerges from this simulation experiment is that the LGMM estimator tends to reduce the bias of the slope parameter even in the conditionally homoskedastic case (see the first panels of Tables 1 and 2) where the conditioning of the error term on past information appears unnecessary. In the next section, we further investigate this bias reduction property of the LGMM estimator by higher-order asymptotic analysis.

\section{Higher-Order Analysis}

\subsection{Stochastic Expansion in AR(1) Model with IID Errors}

In this section, we undertake a stochastic expansion to study the higher-order properties of the LGMM estimator by specializing the model of interest to an $\mathrm{AR}(1)$ process with iid errors. There are two main reasons for this simplification. First, it is well documented that this model could cause a large estimation bias as the persistence of the process increases and the bias properties of

conditional moment restriction $E\left[r_{t}\left(r_{t+1}-\theta_{0} r_{t}\right)\right]=0$. The GLS and other semi-parametrically efficient estimators utilize all information from the conditional moment restriction $E\left[r_{t+1}-\theta_{0} r_{t} \mid r_{t}\right]=0$ to estimate $\theta_{0}$. Thus, these two approaches provide two extreme treatments of the information contained in the conditional moment, and other non-local GMM estimators based on the unconditional moment restriction $E\left[v\left(r_{t}\right)\left(r_{t+1}-\theta_{0} r_{t}\right)\right]=0$ with some vector of instruments $v\left(r_{t}\right)$ can be considered as intermediate cases. 
the OLS estimator are analytically derived and widely discussed in the literature (Marriott and Pope, 1954; Kendall, 1954; among others). Second, the iid assumption on the errors simplifies the LGMM objective function and allows us to focus on a closed form expression for the LGMM estimator. Interestingly, our simulation results reported below suggest that the LGMM estimator enjoys smaller bias (and MSE) than the OLS estimator even when conditioning (or smoothing) is unnecessary due to the iid structure of the errors. This result bears some resemblance to the bias reduction of the smoothed generalized empirical likelihood estimator in serially uncorrelated models shown by Anatolyev (2005) although it is derived in a completely different context and framework.

Suppose that the data are generated by a zero-mean $\mathrm{AR}(1)$ model

$$
r_{t+1}=\theta_{0} r_{t}+u_{t+1}
$$

for each $t=1, \ldots, T$, where $u_{t} \sim i i d(0,1)$, and the conditional moment restriction (1) can be obtained by defining $u\left(y_{t+1}, \theta_{0}\right)=r_{t+1}-\theta_{0} r_{t}$ with $y_{t+1}=\left(r_{t+1}, r_{t}\right)^{\prime}$ and $x_{t}=r_{t} .{ }^{10}$ The case with deterministic terms can be analyzed by decomposing the lagged and deterministic regressors as in van Giersbergen (2005) although the possible bias reduction of the LGMM estimator are expected to come only from the localized weighting of the stochastic components.

To highlight the effect of smoothing on the moment functions, we compare the OLS estimator $\hat{\theta}_{O L S}=\arg \min _{\theta \in \Theta} \sum_{t=1}^{T-1} u\left(y_{t+1}, \theta\right)^{2}$ and the LGMM estimator with a constant weight matrix

$$
\hat{\theta}_{L G M M 1}=\arg \min _{\theta \in \Theta} \sum_{t=1}^{T-1} \mathbb{I}_{t T} u_{T}\left(x_{t}, \theta\right)^{2}=\arg \min _{\theta \in \Theta} \sum_{t=1}^{T-1} \mathbb{I}_{t T}\left[\sum_{j=1}^{T-1} w_{t j}\left(r_{j+1}-\theta r_{j}\right)\right]^{2} .
$$

Note that the difference between $\hat{\theta}_{L G M M 1}$ and $\hat{\theta}_{O L S}$ is whether we smooth the moment function $u\left(y_{t+1}, \theta\right)$ or not. One convenient feature of $\hat{\theta}_{L G M M 1}$ is that it is written by the explicit form

$$
\hat{\theta}_{L G M M 1}=\frac{\sum_{t=1}^{T-1} \mathbb{I}_{t T}\left[\sum_{j=1}^{T-1} w_{t j} r_{j}\right]\left[\sum_{j=1}^{T-1} w_{t j} r_{j+1}\right]}{\sum_{t=1}^{T-1} \mathbb{I}_{t T}\left[\sum_{j=1}^{T-1} w_{t j} r_{j}\right]^{2}}=\theta_{0}+\frac{\sum_{t=1}^{T-1} \mathbb{I}_{t T} \hat{r}_{t} \hat{u}_{t+1}}{\sum_{t=1}^{T-1} \mathbb{I}_{t T} \hat{r}_{t}^{2}},
$$

where $\hat{r}_{t}=\sum_{j=1}^{T-1} w_{t j} r_{j}$ and $\hat{u}_{t+1}=\sum_{j=1}^{T-1} w_{t j} u_{j+1}$.

For the OLS estimator, an analogous expression to (9) is obtained as

$$
\hat{\theta}_{O L S}=\theta_{0}+\frac{\sum_{t=1}^{T-1} r_{t} u_{t+1}}{\sum_{t=1}^{T-1} r_{t}^{2}}
$$

\footnotetext{
${ }^{10}$ The focus of the higher-order analysis here is to gain some insights about the bias properties of the LGMM and OLS estimators in a simple setup and does not target the asymptotically efficient estimator. Thus, we do not augment the conditional moment restriction $E\left[u\left(y_{t+1}, \theta_{0}\right) \mid x_{t}\right]=0$ with the homoskedasticity assumption $E\left[u\left(y_{t+1}, \theta_{0}\right)^{2} \mid x_{t}\right]=1$ when we estimate $\theta_{0}$. The homoskedasticity assumption is only used to simplify the higherorder analysis and make the comparison of the LGMM and OLS more intuitive.
} 
Let $s_{r}^{2}=\frac{1}{T} \sum_{t=1}^{T-1} r_{t}^{2}$ and $\sigma_{r}^{2}=E\left[r_{t-1}^{2}\right]=\frac{1}{1-\theta_{0}^{2}}$. An expansion of $\left(\hat{\theta}_{O L S}-\theta_{0}\right)$ around $s_{r}^{2}=\sigma_{r}^{2}$ yields ${ }^{11}$

$$
\hat{\theta}_{O L S}-\theta_{0}=A_{O L S}-B_{O L S}+O_{p}\left(T^{-3 / 2}\right)
$$

where

$$
A_{O L S}=\frac{1}{\sigma_{r}^{2}} \frac{1}{T} \sum_{t=1}^{T-1} r_{t} u_{t+1}, \quad B_{O L S}=\frac{s_{r}^{2}-\sigma_{r}^{2}}{\sigma_{r}^{4}} \frac{1}{T} \sum_{t=1}^{T-1} r_{t} u_{t+1} .
$$

Note that $E\left[A_{O L S}\right]=0$ in model (7). Simple algebraic manipulations (see Davidson, 2000, for example) yield

$$
E\left[B_{O L S}\right]=\frac{2 \theta_{0}}{T} .
$$

Therefore, as derived in Marriott and Pope (1954) and Kendall (1954), the higher-order bias of the OLS estimator under the model (7) can be expressed as

$$
E\left[\hat{\theta}_{O L S}\right]-\theta_{0}=-\frac{2 \theta_{0}}{T}+O\left(T^{-3 / 2}\right) .
$$

This expansion suggests that the OLS estimator tends to have a negative finite sample bias when $\theta_{0}$ is positive.

We now derive a stochastic expansion of the LGMM estimator with the unit weight matrix $\hat{\theta}_{L G M M 1}$. Recall that $f(\cdot)$ is the marginal density function of $x_{t}=r_{t}$, and denote

$$
\begin{aligned}
f_{t} & =f\left(r_{t}\right), \quad \tilde{u}_{t+1}=\frac{1}{T h} \sum_{j=1}^{T-1} \mathbb{K}\left(\frac{r_{t}-r_{j}}{h}\right) u_{j+1}, \\
\tilde{f}_{t} & =\frac{1}{T h} \sum_{j=1}^{T-1} \mathbb{K}\left(\frac{r_{t}-r_{j}}{h}\right), \quad \bar{f}_{t}=\frac{1}{h} \int \mathbb{K}\left(\frac{r_{t}-r}{h}\right) f(r) d r, \\
\tilde{r}_{t} & =\frac{1}{T h} \sum_{j=1}^{T-1} \mathbb{K}\left(\frac{r_{t}-r_{j}}{h}\right) r_{j}, \quad \bar{r}_{t}=\frac{1}{h} \int \mathbb{K}\left(\frac{r_{t}-r}{h}\right) r f(r) d r, \\
V_{f, t} & =\tilde{f}_{t}-\bar{f}_{t}, \quad B_{f, t}=\bar{f}_{t}-f_{t}, \quad V_{r, t}=\tilde{r}_{t}-\bar{r}_{t}, \quad B_{r, t}=\bar{r}_{t}-r_{t} .
\end{aligned}
$$

Based on this notation, we make the following assumptions.

Assumption A4. Assume that

(i) $E\left|u_{t}\right|^{4}<\infty$ and $E\left[f_{t}^{-8}\right]<\infty$ for each $t=1, \ldots, T$.

(ii) There exists a positive sequence $\left\{a_{T}\right\}_{T \geq 1}$ such that $a_{T} \rightarrow 0$ and $T^{1 / 2} a_{T} \rightarrow \infty$ as $T \rightarrow \infty$, and

$$
\sup _{\left\{t: \mathbb{I}_{t T}=1\right\}}\left|\tilde{u}_{t}\right|=O_{p}\left(a_{T}\right), \quad \sup _{\left\{t: \mathbb{I}_{t T}=1\right\}}\left|V_{f, t}\right|=O_{p}\left(a_{T}\right), \sup _{\left\{t: \mathbb{I}_{t T}=1\right\}}\left|V_{r, t}\right|=O_{p}\left(a_{T}\right) .
$$

\footnotetext{
${ }^{11}$ The remainder term $O_{p}\left(T^{-3 / 2}\right)$ follows from $\frac{1}{T} \sum_{t=1}^{T} r_{t} u_{t+1}=O_{p}\left(T^{-1 / 2}\right)$ and $s_{r}^{2}-\sigma_{r}^{2}=O_{p}\left(T^{-1 / 2}\right)$ by the central limit theorem.
} 
(iii) There exists a positive sequence $\left\{b_{T}\right\}_{T \geq 1}$ such that $b_{T} \rightarrow 0$ and $T^{1 / 2} b_{T} \rightarrow \infty$ as $T \rightarrow \infty$, and

$$
\sup _{\left\{t: \mathbb{I}_{t T}=1\right\}}\left|B_{f, t}\right|=O\left(b_{T}\right), \sup _{\left\{t: \mathbb{I}_{t T}=1\right\}}\left|B_{r, t}\right|=O\left(b_{T}\right) .
$$

Assumption A4 (i) is concerned with the existence of higher-order moments. These assumptions are used to derive the law of large numbers, such as $\frac{1}{T} \sum_{t=1}^{T-1}\left|\frac{r_{t}^{2}}{f_{t}^{4}}\right| \stackrel{p}{\rightarrow} E\left|\frac{r_{t}^{2}}{f_{t}^{4}}\right|<\infty$ to guarantee $\frac{1}{T} \sum_{t=1}^{T-1} \mathbb{I}_{t T}\left|\frac{r_{t}^{2}}{f_{t}^{4}}\right|=O_{p}(1)$. Assumption A4 (ii) and (iii) contain higher level conditions for the variance and bias components of the kernel estimators, respectively. Lemma A in Appendix A provides primitive conditions that guarantee the validity of these assumptions. Let $\tilde{s}_{r}^{2}=\frac{1}{T} \sum_{t=1}^{T-1} \mathbb{I}_{t T} r_{t}^{2}$. Based on these assumptions, we obtain the following stochastic expansion of $\hat{\theta}_{L G M M 1}$.

Theorem 2. Under the model (7) and Assumption A4,

$$
\hat{\theta}_{L G M M 1}-\theta_{0}=A_{L G M M}-B_{L G M M}+C_{L G M M}+D_{L G M M}+O_{p}\left(a_{T}\left(a_{T}+b_{T}\right)^{2}\right),
$$

where

$$
\begin{aligned}
A_{L G M M} & =\frac{1}{\sigma_{r}^{2}} \frac{1}{T} \sum_{t=1}^{T-1} \mathbb{I}_{t T} \frac{r_{t} \tilde{u}_{t+1}}{f_{t}}, \quad B_{L G M M}=\frac{\tilde{s}_{r}^{2}-\sigma_{r}^{2}}{\sigma_{r}^{4}} \frac{1}{T} \sum_{t=1}^{T-1} \mathbb{I}_{t T} \frac{r_{t} \tilde{u}_{t+1}}{f_{t}}, \\
C_{L G M M} & =\frac{1}{\sigma_{r}^{2}} \frac{1}{T} \sum_{t=1}^{T-1} \mathbb{I}_{t T} \frac{\left(V_{r, t}+B_{r, t}\right) \tilde{u}_{t+1}}{f_{t}^{2}}-\frac{2}{\sigma_{r}^{4}} \frac{1}{T} \sum_{t=1}^{T-1} \mathbb{I}_{t T} \frac{r_{t}\left(V_{r, t}+B_{r, t}\right)}{f_{t}} \frac{1}{T} \sum_{t=1}^{T-1} \mathbb{I}_{t T} \frac{r_{t} \tilde{u}_{t+1}}{f_{t}}, \\
D_{L G M M} & =-\frac{2}{\sigma_{r}^{2}} \frac{1}{T} \sum_{t=1}^{T-1} \mathbb{I}_{t T} \frac{\left(V_{f, t}+B_{f, t}\right) r_{t} \tilde{u}_{t+1}}{f_{t}^{2}}+\frac{2}{\sigma_{r}^{4}} \frac{1}{T} \sum_{t=1}^{T-1} \mathbb{I}_{t T} \frac{r_{t}^{2}\left(V_{f, t}+B_{f, t}\right)}{f_{t}} \frac{1}{T} \sum_{t=1}^{T-1} \mathbb{I}_{t T} \frac{r_{t} \tilde{u}_{t+1}}{f_{t}} .
\end{aligned}
$$

We first check the stochastic orders of these terms. The first term $A_{L G M M}$ corresponds to $A_{O L S}$ in (10). By applying a $U$-statistic argument as in the proof of Theorem 1 (b), we typically have $A_{L G M M}=O_{p}\left(T^{-1 / 2}\right)$. The second term $B_{L G M M}$ corresponds to $B_{O L S}$ in (10). Since $\tilde{s}_{r}^{2}-\sigma_{r}^{2}=$ $O_{p}\left(T^{-1 / 2}\right)$ and $\frac{1}{T} \sum_{t=1}^{T-1} \mathbb{I}_{t T} \frac{r_{t} \tilde{u}_{t+1}}{f_{t}}=O_{p}\left(T^{-1 / 2}\right)$ by the central limit theorem and a $U$-statistic argument, respectively, we typically have $B_{L G M M}=O_{p}\left(T^{-1}\right)$. The third term $C_{L G M M}$ arises from the correlation among $V_{r, t}+B_{r, t}, \tilde{u}_{t+1}$, and $r_{t}$. Under Assumption A4, this term satisfies $C_{L G M M}=O_{p}\left(a_{T}\left(a_{T}+b_{T}\right)\right)$. Similarly, the fourth term $D_{L G M M}$ arises from the correlation among $V_{f, t}+B_{f, t}, \tilde{u}_{t+1}$, and $r_{t}$, and satisfies $D_{L G M M}=O_{p}\left(a_{T}\left(a_{T}+b_{T}\right)\right)$ under Assumption A4.

Based on the stochastic orders of these terms, we focus on the dominant term $A_{L G M M}$ and compare it with the OLS counterpart $A_{O L S}$. For an intuitive argument, let us neglect the trimming term $\mathbb{I}_{t T}$ in $A_{L G M M}$ and consider $A_{L G M M}^{*}=\frac{1}{\sigma_{r}^{2}} \frac{1}{T} \sum_{t=1}^{T-1} \frac{r_{t} \tilde{u}_{t+1}}{f_{t}}$. Provided that $\sup _{1 \leq t \leq T}\left|\mathbb{I}_{t T}-1\right|$ converges to zero sufficiently fast (which is guaranteed if $c_{T} \propto T^{\xi}$ and $E\left|x_{t}\right|^{\zeta}<\infty$ for sufficiently large $\xi$ and $\zeta), A_{L G M M}^{*}$ can serve as a reasonable approximation to $A_{L G M M}$. The expectation $E\left[A_{L G M M}^{*}\right]$ can be approximated as follows. Let $f_{u}(\cdot)$ be the density function of $u_{t}$. 
Theorem 3. Suppose that the model (7) and Assumptions A2 (iv) and A3 hold. Then,

$$
E\left[A_{L G M M}^{*}\right]=\frac{1-\theta_{0}^{2}}{T^{2}} \sum_{t=1}^{T-1}\left[\frac{1}{\left(1-\theta_{0}\right)^{2}}+\frac{\theta_{0}}{\left(1-\theta_{0}^{2}\right)^{2}}+\cdots+\frac{\theta_{0}^{t-2}}{\left(1-\theta_{0}^{t-1}\right)^{2}}\right]+O\left(T^{-1} h^{\omega}\right) .
$$

Note that in contrast to $E\left[A_{O L S}\right]=0$, the expectation $E\left[A_{L G M M}^{*}\right]$ tends to be positive and is of order $O\left(T^{-1}\right)$ when $0 \leq \theta_{0}<1$. This is expected since the weighting scheme uses information from neighboring observations and destroys the zero correlation between $r_{t}$ and $u_{t}$. Interestingly, this proves to be advantageous for the LGMM estimator since the positive value of $E\left[A_{L G M M}^{*}\right]$ tends to offset the second bias term $E\left[B_{L G M M}\right]$ in the expansion. Also, it is worth noting that despite the fact that the bandwidth parameter has only a second-order effect on the magnitude of $E\left[A_{L G M M}^{*}\right]$, a data-driven (simulation- or bootstrap-based) choice of $h$ can be used to reduce or even completely eliminate the $O\left(T^{-1}\right)$ terms in the bias expansion.

Although it is difficult to evaluate the second term $E\left[B_{L G M M}\right]$ without specifying the distributional form of $f_{u}$, the stochastic order $B_{L G M M}=O_{p}\left(T^{-1}\right)$ implies $E\left[B_{L G M M}\right]=O\left(T^{-1}\right)$ if $B_{L G M M}$ is uniformly integrable. Thus, as long as $E\left[B_{L G M M}\right]$ shows a similar behavior as $E\left[B_{O L S}\right]$ in finite samples, the positive bias term $E\left[A_{L G M M}^{*}\right]$ may compensate for the negative bias caused by $E\left[B_{L G M M}\right]$. We evaluate this conjecture by simulation in the next subsection.

\subsection{Performance of LGMM Estimator with Unit Weight}

To assess the finite-sample properties of the LGMM estimator with the unit weight in models with iid errors, we conduct a small Monte Carlo experiment. The data are generated from the zero-mean $\mathrm{AR}(1)$ model

$$
r_{t+1}=\theta_{0} r_{t}+u_{t+1}
$$

for each $t=1, \ldots, T$, where $u_{t+1} \sim i i d N(0,1)$ and $\theta_{0}=0.95$. As in Section 3.2, the weights for the LGMM estimator are obtained from the Gaussian kernel and plug-in bandwidth and the results are based on 5,000 Monte Carlo replications. Unlike the experiment in Section 3.2, however, the zero intercept is imposed in the estimation in order to be consistent with the theoretical framework adopted above. The mean bias, standard deviation and RMSE of the three estimators are reported in Table 3 for sample sizes $T=50,100,200,400,800,1600$, and 3200 .

To illustrate the source of the bias reduction in the LGMM estimator, we also numerically evaluate the two leading terms in the bias expansion for the OLS and LGMM estimators. In particular, the last two columns of Table 3 report the Monte Carlo averages of $\frac{1}{\sigma_{r}^{2}} \frac{1}{T} \sum_{t=1}^{T-1} r_{t} u_{t+1}$ (to evaluate $E\left[A_{O L S}\right]$ ) and $\frac{s_{r}^{2}-\sigma_{r}^{2}}{\sigma_{r}^{4}} \frac{1}{T} \sum_{t=1}^{T-1} r_{t} u_{t+1}$ (to evaluate $E\left[B_{O L S}\right]$ ) regarding the OLS estimator and $\frac{1}{\sigma_{r}^{2}} \frac{1}{T} \sum_{t=1}^{T-1} r_{t}\left(\tilde{u}_{t+1} / f_{t}\right)$ (to evaluate $E\left[A_{L G M M}\right]$ ) and $\frac{\tilde{s}_{r}^{2}-\sigma_{r}^{2}}{\sigma_{r}^{4}} \frac{1}{T} \sum_{t=1}^{T-1} r_{t}\left(\tilde{u}_{t+1} / f_{t}\right)$ (to evaluate $\left.E\left[B_{L G M M}\right]\right)$ regarding the LGMM estimator. 
The numerical results in Table 3 support our theoretical results in the previous subsection. The LGMM enjoys a substantially smaller mean bias which goes to zero much faster than the OLS estimator. The bias reduction property of LGMM along with its efficiency result in a lower RMSE for small sample sizes although the difference in RMSE converges to zero as the sample size increases. ${ }^{12}$ As conjectured above, the terms $E\left[B_{O L S}\right]$ and $E\left[B_{L G M M}\right]$ for the OLS and LGMM estimators are of similar magnitude and the better bias properties of the LGMM estimator arise from the fact that the positive value of $E\left[A_{L G M M}\right]$ tends to offset the downward bias effect of $E\left[B_{L G M M}\right]$.

In order to visualize the bias of the LGMM estimator over the stationary region of the AR parameter space, Figure 1 plots the bias functions of the OLS and LGMM estimators for $\theta_{0}=$ $-0.95,-0.85, \ldots, 0.85,0.95$ for two sample sizes: $T=100$ and 200. As our higher-order analysis in Section 4.1 suggests, the bias reduction property of the LGMM estimator is not expected to hold over the whole parameter space. For example, when $-1<\theta_{0}<0$, the sign of $E\left[A_{L G M M}^{*}\right]$ in (13) cannot be determined unambiguously since the terms inside the square brackets of (13) are of alternating signs. For this reason, it would be interesting to see how the LGMM estimator performs over the negative part of the parameter space even though our main interest lies in positively autocorrelated processes.

To explore the effect of the smoothing parameter choice on the LGMM estimator, we present the results for three bandwidths: $h=T^{-1 / 5}$ (approximate iid bandwidth), $h=0.6 T^{-1 / 5}$ and $h=1.2 T^{-1 / 5}$ with a standardized conditioning variable. The mean bias is computed as a Monte Carlo average over 100,000 replications. Figure 1 shows that while the OLS bias increases linearly with the absolute value of $\theta_{0}$ as expression (11) suggests, the bias function of the LGMM estimator appears to flatten out for $\left|\theta_{0}\right| \geq 0.5$. For $h=T^{-1 / 5}$ and $h=1.2 T^{-1 / 5}$, the bias of the LGMM estimator is substantially smaller than the OLS bias for $\theta_{0} \geq 0.2$ and $\theta_{0} \leq-0.6$ although the LGMM estimators with these bandwidths exhibit positive bias for $\theta_{0}$ less than 0.2 . While the LGMM bias exceeds the OLS bias over a part of the negative region $(-0.6,0)$, the difference is relatively small. As expected, the bias of the LGMM estimator approaches the OLS bias as the bandwidth gets smaller.

In the positive part of the parameter space, which is of primary interest for our analysis, the LGMM demonstrates convincingly its higher-order advantages and appears practically unbiased for $T=200, \theta_{0} \in[0.2,0.7]$ and $h=1.2 T^{-1 / 5}$. Finally, Figure 1 clearly suggests that the different levels of persistence seem to require different bandwidths (smaller bandwidths when $\theta_{0}$ is near zero and

\footnotetext{
${ }^{12}$ In an earlier version of the paper, we also included the full maximum likelihood estimator (MLE) that incorporates information about the first observation in the likelihood function (Beach and MacKinnon, 1978). The standard deviation of the LGMM estimator was only slightly higher than the full MLE for all sample sizes but the LGMM estimator dominated the MLE in terms of RMSE. These results are available from the authors upon request.
} 
larger bandwidths when $\theta_{0}$ is near one) and a data-driven procedure for selecting the appropriate smoothing parameter would prove beneficial.

\section{Economic Significance of LGMM}

To evaluate the economic significance of the statistical properties of the LGMM estimator, we use this estimator for bond and derivative pricing with data generated from the CIR (Cox, Ingersoll and Ross, 1985) model

$$
d r_{t}=\kappa\left(\mu-r_{t}\right) d t+\sigma r_{t}^{1 / 2} d W_{t}
$$

for each $t=0,1, \ldots, T$. This model is convenient because the transition and marginal densities are known and the bond and call option prices are available in closed form (Cox, Ingersoll and Ross, 1985). 5,000 sample paths for the spot interest rate of length $T=600$ observations are simulated using the procedure described in Chapman and Pearson (2000). After drawing an initial value from the marginal Gamma density, the interest rate process is constructed recursively by drawing random numbers from the transition non-central chi-square density and using the values for $\kappa, \mu$ and $\sigma$ and a time step between two consecutive observation equal to $\Delta=1 / 52$ that corresponds to weekly data. Carrasco, Chernov, Florens and Ghysels (2007) show that the CIR process (14) is absolutely regular.

We consider one of the parameter configurations that are used in Chapman and Pearson (2000) and are calibrated to the interest rate data in Aït-Sahalia (1996). ${ }^{13}$ In particular, $(\kappa, \mu, \sigma)=$ $(0.21459,0.085711,0.0783)$ which implies a highly persistent interest rate process consistent with the observed data. The expressions for the price of a zero-coupon discount bond and a call option on a zero-coupon discount bond have an analytical form and are given in Cox, Ingersoll and Ross (1985). We follow Phillips and Yu (2005) and compute the prices of a three-year zero-coupon discount bond and a one-year European call option on a three-year discount bond with a face value of $\$ 100$ and an exercise price of $\$ 87$ given an initial interest rate of $5 \%$. The bond and option prices are computed assuming that the market price of risk is equal to zero.

The parameters of model (3) are typically estimated from the discrete-time representation $u_{t+1}=\Delta r_{t+1}-\alpha_{0}-\alpha_{1} r_{t}$. The GMM estimator based on the unconditional moment restrictions $E\left[u_{t+1}\right]=0, E\left[u_{t+1} r_{t}\right]=0$ and $E\left[u_{t+1}^{2}\right]=\sigma^{2} r_{t}$ is the GLS estimator used in Ball and Torous (1996) and Chapman and Pearson (2000). In addition to the GLS estimator, we consider the efficient estimator of Carrasco and Florens (2000) with the same smoothing parameter and integrating den-

\footnotetext{
${ }^{13}$ The results from the second parametrization in Chapman and Person (2000) are qualitatively similar and are not reported to preserve space. These results and some additional simulations for the specifications used in Phillips and $\mathrm{Yu}(2005)$ are available from the authors upon request.
} 
sity as in Section 3.2. ${ }^{14}$ Note also that because the transition and marginal densities for the CIR model are available in closed form, one could perform exact maximum likelihood estimation as in Phillips and Yu (2005) and avoid the discretization bias. Since Phillips and Yu (2005) found that the effects of the discretization bias are small, we do not consider explicitly the maximum likelihood estimator and focus only on the GMM estimators. ${ }^{15}$ Similarly, we do not assume knowledge of the characteristic function (as in Carrasco, Chernov, Florens and Ghysels, 2007) in constructing the moment conditions for the Carrasco and Florens (2000) estimator. As a result, in estimating the parameters $\alpha_{0}$ and $\alpha_{1}$ (or equivalently, $\kappa$ and $\mu$ ), both the LGMM and the Carrasco-Florens estimators are based on the conditional moment restriction $E\left[u_{t+1} \mid r_{t}\right]=0$. The unconditional variance $\sigma^{2}$ for LGMM and Carrasco-Florens estimators is then computed as a sample average of the corresponding squared residuals standardized by $r_{t}$ and adjusted for $\triangle$.

Table 4 reports the median, mean, standard deviation and root mean square error (RMSE) of the parameter estimates, bond and option prices. Due to the strong persistence of the data and the autoregressive structure of the discretized model, the parameter that measures the speed of mean reversion is estimated with a substantial upward bias. Since the option price is very sensitive to this parameter, the bias of the estimate of $\kappa$ is translated into a downward bias in the option price which is further exacerbated by the highly nonlinear relationship between the option price and $\kappa$. While all estimators of $\kappa$ are biased, the bias of the GLS and Carrasco-Florens estimators is twice as large as the bias of the conditional GMM estimator. Interestingly, the variance of the LGMM estimator of $\kappa$ is approximately 30\% smaller than the variance of the GLS and Carrasco-Florens estimators. This is typically not the case for some popular bias reduction techniques such as simulation-based and jackknife methods where the bias correction is accompanied with a higher variability of the estimates. As a result of the lower bias and increased efficiency, the RMSE of the LGMM, obtained as the square root of the sum of the squared mean bias and variance of the estimator, is more than $30 \%$ smaller that the RMSE of the other two estimators.

Similar results emerge from the estimates of the intercept in the model. In this case, the RMSE of the LGMM estimator is 32-36\% lower than the RMSE of the other estimators. The performance of the LGMM estimator is even more impressive given the fact that, unlike the GLS, it does not utilize any knowledge of the conditional variance function and should be expected to possess some robustness in this respect.

Despite the excellent properties of the LGMM estimator of the intercept and slope parameters

\footnotetext{
${ }^{14}$ The results from the optimal instrumental variables estimator of Kuersteiner (2002) are not presented because the numerical performance of this estimator turned out to be highly unstable in our highly persistent setup and was dominated by the other estimators.

${ }^{15}$ While the applicability of the ML approach is limited by the fact that the likelihood function is available in closed form only for the CIR specification, the GMM estimators can accommodate a much larger class of models. Most importantly, the LGMM estimator does not require any knowledge of the parametric form of the diffusion function.
} 
in the discrete-time AR model, the long-run mean $\mu$ in (14) is computed as a ratio of two estimates and exhibits some instability when $\alpha_{1}$ (or equivalently $\kappa$ ) is close to 0. Since the LGMM estimator is less biased than the other estimators, it tends to produce more values of $\alpha_{1}$ near 0 which leads to the higher variability of this estimator in Table 4. Similar findings have been reported by Ball and Torous (1996) who point out that this instability is particularly pronounced at low levels of the initial interest rate. Finally, the unconditional standard deviation of the error component is estimated with a slight upward bias and the differences in the performance of the estimators for this parameter are negligible.

The results for the bond and call option prices obtained by plugging the estimated parameters from the different methods are reported in the last two columns of Table 4 . The bond price computed from the LGMM estimator appears to be the least biased and most efficient with a RMSE which is $20 \%$ lower than the RMSEs of the other two estimators. As pointed out above, the price of the call option is more sensitive to the CIR model parameters and the economic significance of the effects of the lower bias of the LGMM estimator is more evident. For instance, for the true option price of $\$ 1.931$, the average LGMM-based option price is $\$ 1.652$ compared to $\$ 1.110$ and $\$ 1.127$ for Carrasco-Florens and GLS estimator, respectively. The higher variability of the LGMM option price is due to two reasons. First, it partly arises from the higher variability of the estimate of $\mu$ which was caused by the near unit root specification of the interest rate process. More importantly, however, the lower variability of the other two option prices is somewhat artificial and is due to a large number of economically unreasonable option values around zero. Overall, the attractive properties of the LGMM estimator translate into large gains for pricing bonds and call options.

\section{Conclusion}

This paper studies the properties of the local GMM estimator in Markov models with conditional heteroskedasticity. We derive the conditions for the consistency, asymptotic normality, and semiparametric efficiency of the local GMM estimator under this time series setup. The paper also undertakes a higher-order analysis based on an asymptotic expansion to study the bias properties of the local GMM estimator. Some interesting findings about the bias structure and the order of magnitudes of the leading terms in the expansion emerge from this analysis. The finite-sample performance of the LGMM estimator is evaluated in the context of bond and derivative pricing with simulated data from a diffusion model of spot interest rate. In summary, the semi-parametric efficiency, smaller bias, and computational simplicity of the local GMM estimator prove to be very desirable and appealing properties from both theoretical and practical perspectives for estimating time series models defined by conditional moment restrictions.

This paper can be considered as a starting point toward the goal of better understanding the 
finite-sample properties of the LGMM estimator for general time series models. Several natural extensions of our results on the bias properties of the unit weight LGMM in AR(1) models include higher-order analyses of (i) optimally weighted LGMM estimator, (ii) times series models with more general dependence structure, and (iii) variance of the LGMM estimator. Since these extensions require rather different and advanced technical arguments, we leave the analysis of these interesting issues for future research. 


\section{A Appendix: Derivations and Mathematical Proofs}

Hereafter, let $\hat{\theta}=\hat{\theta}_{L G M M}$, "sup $"$ " denote "sup $\operatorname{su}_{t \in\left\{t: \mathbb{I}_{t T}=1\right\}}$ ", and "w.p.a.1" signify "with probability approaching one." We repeatedly use the following uniform convergence result by Kristensen (2009, Theorem 1).

Lemma A. Suppose Assumptions A1, A2 (i), and A3 (i) hold and the function $a: \mathbb{R}^{p+1} \times A$ is $\mathcal{D}$-bounded on $A$ with order $s>2$. If $\log T /\left(T h^{p}\right) \rightarrow 0$ as $T \rightarrow \infty$, then for any $0<\xi<\infty$,

$\sup _{|x| \leq c_{T}} \sup _{\theta \in A}\left|\frac{1}{(T-p) h^{p}} \sum_{j=p}^{T-1} \mathbb{K}\left(\frac{x_{j}-x}{h}\right) a\left(y_{j+1}, \theta\right)-E\left[\frac{1}{h^{p}} \mathbb{K}\left(\frac{x_{j}-x}{h}\right) a\left(y_{j+1}, \theta\right)\right]\right|=O_{p}\left(\sqrt{\frac{\log T}{T h^{p}}}\right)$ with $c_{T} \propto T^{\xi}$.

\section{A.1 Proof of Theorem 1}

Proof of Part (a): The objective function of the LGMM estimator and its population counterpart are written respectively as

$$
\begin{aligned}
Q_{T}(\theta) & =\frac{1}{T-p} \sum_{t=p}^{T-1} \mathbb{I}_{t T} u_{T}\left(x_{t}, \theta\right)^{\prime} V_{T}\left(x_{t}, \theta\right)^{-1} u_{T}\left(x_{t}, \theta\right), \\
Q(\theta) & =E\left[E\left[u\left(y_{t+1}, \theta\right) \mid x_{t}\right]^{\prime} V\left(x_{t}, \theta\right)^{-1} E\left[u\left(y_{t+1}, \theta\right) \mid x_{t}\right]\right],
\end{aligned}
$$

where $V(x, \theta)^{-1}$ exists for each $x \in \mathbb{R}^{p}$ and $\theta \in \Theta$ from Assumption A2 (iii), and $V_{T}\left(x_{t}, \theta\right)^{-1}$ exists w.p.a.1 for each $t \in\left\{t: \mathbb{I}_{t T}=1\right\}$ and $\theta \in \Theta$ from Assumption A2 (iii) and (16) below. From Newey and McFadden (1994, Theorem 2.1), it is sufficient to show that

(i) $\Theta$ is compact (assumed in Assumption A2 (i)),

(ii) $Q(\theta)$ is uniquely minimized at $\theta_{0}$ (implied by Assumption A2 (ii)),

(iii) $Q(\theta)$ is continuous on $\Theta$ (implied by Assumption A2 (iii)),

(iv) $\sup _{\theta \in \Theta}\left|Q_{T}(\theta)-Q(\theta)\right| \stackrel{p}{\rightarrow} 0$.

Thus, it remains to show (iv), which follows from

$$
\begin{aligned}
& \sup _{t} \sup _{\theta \in \Theta}\left|u_{T}\left(x_{t}, \theta\right)-E\left[u\left(y_{t+1}, \theta\right) \mid x_{t}\right]\right| \stackrel{p}{\rightarrow} 0, \\
& \sup _{t} \sup _{\theta \in \Theta}\left|V_{T}\left(x_{t}, \theta\right)-V\left(x_{t}, \theta\right)\right| \stackrel{p}{\rightarrow} 0, \\
& \sup _{\theta \in \Theta}\left|\frac{1}{T-p} \sum_{t=p}^{T-1} \mathbb{I}_{t T} E\left[u\left(y_{t+1}, \theta\right) \mid x_{t}\right]^{\prime} V\left(x_{t}, \theta\right)^{-1} E\left[u\left(y_{t+1}, \theta\right) \mid x_{t}\right]-Q(\theta)\right| \stackrel{p}{\rightarrow} 0 .
\end{aligned}
$$


To show (15), define $\hat{f}(x)=\frac{1}{(T-p) h^{p}} \sum_{j=p}^{T-1} \mathbb{K}\left(\frac{x_{j}-x}{h}\right)$ and $\hat{u}(x, \theta)=\frac{1}{(T-p) h^{p}} \sum_{j=p}^{T-1} \mathbb{K}\left(\frac{x_{j}-x}{h}\right) u\left(y_{j+1}, \theta\right)$. From Lemma A with $a\left(y_{j+1}, \theta\right)=1$ and $u\left(y_{j+1}, \theta\right)$,

$$
\begin{gathered}
\sup _{|x| \leq c_{T}}|\hat{f}(x)-E[\hat{f}(x)]|=O_{p}\left(\sqrt{\frac{\log T}{T h^{p}}}\right), \\
\sup _{|x| \leq c_{T}} \sup _{\theta \in \Theta}|\hat{u}(x, \theta)-E[\hat{u}(x, \theta)]|=O_{p}\left(\sqrt{\frac{\log T}{T h^{p}}}\right) .
\end{gathered}
$$

By a change of variables $\left(a=\frac{x_{j}-x}{h}\right)$ and an expansion around $a=0$,

$$
\sup _{|x| \leq c_{T}}|E[\hat{f}(x)]-f(x)|=h^{p} \sup _{|x| \leq c_{T}}\left|\int \mathbb{K}(a) \frac{d f(x+\bar{a} h)}{d a} d a\right|=O\left(h^{p}\right),
$$

where $\bar{a}$ is a point on the line joining $a$ and 0 . A similar argument with the law of iterated expectations yields

$$
\sup _{|x| \leq c_{T}} \sup _{\theta \in \Theta}\left|E[\hat{u}(x, \theta)]-E\left[u\left(y_{t+1}, \theta\right) \mid x_{t}=x\right] f(x)\right|=O\left(h^{p}\right) .
$$

Combining these results, we obtain

$$
\begin{gathered}
\sup _{|x| \leq c_{T}}|\hat{f}(x)-f(x)|=O_{p}\left(\sqrt{\frac{\log T}{T h^{p}}}\right)+O\left(h^{p}\right), \\
\sup _{|x| \leq c_{T}} \sup _{\theta \in \Theta}\left|\hat{u}(x, \theta)-E\left[u\left(y_{t+1}, \theta\right) \mid x_{t}=x\right] f(x)\right|=O_{p}\left(\sqrt{\frac{\log T}{T h^{p}}}\right)+O\left(h^{p}\right) .
\end{gathered}
$$

Since the denominator and numerator of $u_{T}(x, \theta)=\frac{\hat{u}(x, \theta) / f(x)}{\hat{f}(x) / f(x)}$ satisfy

$$
\sup _{|x| \leq c_{T}}\left|\frac{\hat{f}(x)}{f(x)}-1\right| \leq \frac{\sup _{|x| \leq c_{T}}|\hat{f}(x)-f(x)|}{\inf _{|x| \leq c_{T}} f(x)}=O_{p}\left(\sqrt{\frac{\log T}{\delta_{T}^{2} T h^{p}}}\right)+O\left(\frac{h^{p}}{\delta_{T}}\right),
$$

and

$$
\begin{aligned}
\sup _{|x| \leq c_{T}} \sup _{\theta \in \Theta}\left|\frac{\hat{u}(x)}{f(x)}-E\left[u\left(y_{t+1}, \theta\right) \mid x_{t}=x\right]\right| & \leq \frac{\sup _{|x| \leq c_{T}} \sup _{\theta \in \Theta}\left|E[\hat{u}(x, \theta)]-E\left[u\left(y_{t+1}, \theta\right) \mid x_{t}=x\right] f(x)\right|}{\inf _{|x| \leq c_{T}} f(x)} \\
& =O_{p}\left(\sqrt{\frac{\log T}{\delta_{T}^{2} T h^{p}}}\right)+O\left(\frac{h^{p}}{\delta_{T}}\right)
\end{aligned}
$$

Assumption A3 (ii) guarantees that

$$
\sup _{t} \sup _{\theta \in \Theta}\left|u_{T}\left(x_{t}, \theta\right)-E\left[u\left(y_{t+1}, \theta\right) \mid x_{t}\right]\right|=O_{p}\left(\sqrt{\frac{\log T}{\delta_{T}^{2} T h^{p}}}\right)+O\left(\frac{h^{p}}{\delta_{T}}\right) \stackrel{p}{\rightarrow} 0 .
$$

This delivers the desired result in (15). 
By a similar argument, using Lemma A with $a\left(y_{j+1}, \theta\right)=u\left(y_{j+1}, \theta\right) u\left(y_{j+1}, \theta\right)^{\prime}$, we obtain (16). Also, by the law of large numbers and $\sup _{1 \leq t \leq T}\left|\mathbb{I}_{t T}-1\right| \stackrel{p}{\rightarrow} 0$ (by Kitamura, Tripathi and Ahn (2004, Lemma D.3)), we obtain (17). This completes the proof of part (a) in Theorem 1.

Proof of Part (b). By expanding the first-order condition $\partial Q_{T}(\hat{\theta}) / \partial \theta=0$ around $\theta_{0}$,

$$
\frac{\partial Q_{T}\left(\theta_{0}\right)}{\partial \theta}+\frac{\partial^{2} Q_{T}(\bar{\theta})}{\partial \theta \partial \theta^{\prime}}\left(\widehat{\theta}-\theta_{0}\right)=0
$$

where $\bar{\theta}$ is a point on the line joining $\hat{\theta}$ and $\theta_{0}$. It is sufficient for the conclusion to show that

$$
\begin{aligned}
& \frac{\sqrt{T}}{2} \frac{\partial Q_{T}\left(\theta_{0}\right)}{\partial \theta} \stackrel{d}{\rightarrow} N\left(0, \mathcal{I}\left(\theta_{0}\right)\right), \\
& \frac{1}{2} \frac{\partial^{2} Q_{T}(\bar{\theta})}{\partial \theta \partial \theta^{\prime}} \stackrel{p}{\rightarrow} \mathcal{I}\left(\theta_{0}\right) .
\end{aligned}
$$

To show (19), observe that (see, Donald and Newey, 2000)

$$
\begin{aligned}
\frac{\sqrt{T}}{2} \frac{\partial Q_{T}\left(\theta_{0}\right)}{\partial \theta}= & \frac{1}{\sqrt{T}} \sum_{t=p}^{T-1} \mathbb{I}_{t T}\left[\sum_{j=p}^{T-1} w_{t j} \frac{\partial u\left(y_{j+1}, \theta_{0}\right)}{\partial \theta^{\prime}}\right]^{\prime} V_{T}\left(x_{t}, \theta_{0}\right)^{-1} u_{T}\left(x_{t}, \theta_{0}\right) \\
& +\left(A_{2,1}\left(\theta_{0}\right), \ldots, A_{2, k}\left(\theta_{0}\right)\right)^{\prime} \\
= & A_{1}\left(\theta_{0}\right)+A_{2}\left(\theta_{0}\right)
\end{aligned}
$$

w.p.a.1, where $A_{1}\left(\theta_{0}\right)$ and $A_{2}\left(\theta_{0}\right)$ are implicitly defined and

$$
\begin{aligned}
A_{2, l}\left(\theta_{0}\right) & =\frac{1}{2 \sqrt{T}} \sum_{t=p}^{T-1} \mathbb{I}_{t T} u_{T}\left(x_{t}, \theta_{0}\right)^{\prime} \frac{\partial V_{T}\left(x_{t}, \theta_{0}\right)^{-1}}{\partial \theta_{l}} u_{T}\left(x_{t}, \theta_{0}\right), \\
\frac{\partial V_{T}\left(x_{t}, \theta_{0}\right)^{-1}}{\partial \theta_{l}} & =-V_{T}\left(x_{t}, \theta_{0}\right)^{-1} S_{T}\left(x_{t}, \theta_{0}\right) V_{T}\left(x_{t}, \theta_{0}\right)^{-1}, \\
S_{T}\left(x_{t}, \theta_{0}\right) & =\sum_{j=p}^{T-1} w_{t j} u\left(y_{j+1}, \theta_{0}\right)\left(\frac{\partial u\left(y_{j+1}, \theta_{0}\right)}{\partial \theta_{l}}\right)^{\prime}+\sum_{j=p}^{T-1} w_{t j} \frac{\partial u\left(y_{j+1}, \theta_{0}\right)}{\partial \theta_{l}} u\left(y_{j+1}, \theta_{0}\right)^{\prime}
\end{aligned}
$$

for $l=1, \ldots, k$.

We first consider $A_{2}\left(\theta_{0}\right)$. Using a similar argument as in the derivation of (18) with $E\left[u\left(y_{t+1}, \theta_{0}\right) \mid x_{t}=x\right]=$ 0 (Assumption A2 (ii)),

$$
\sup _{t}\left|u_{T}\left(x_{t}, \theta_{0}\right)\right|=O_{p}\left(\sqrt{\frac{\log T}{\delta_{T}^{2} T h^{p}}}\right)=o_{p}\left(T^{-1 / 4}\right),
$$

where the second equality follows from Assumption A3 (ii).

From (16), we have $\sup _{t}\left|V_{T}\left(x_{t}, \theta_{0}\right)-V\left(x_{t}, \theta_{0}\right)\right| \stackrel{p}{\rightarrow} 0$. Applying a similar argument in deriving (18) with Lemma A for $a\left(y_{j+j}, \theta_{0}\right)=\frac{\partial u\left(y_{j+1}, \theta_{0}\right)}{\partial \theta_{l}} u\left(y_{j+1}, \theta_{0}\right)$ yields

$$
\sup _{t}\left|\sum_{j=p}^{T-1} w_{t j} \frac{\partial u\left(y_{j+1}, \theta_{0}\right)}{\partial \theta_{l}} u\left(y_{j+1}, \theta_{0}\right)^{\prime}-E\left[\frac{\partial u\left(y_{t+1}, \theta_{0}\right)}{\partial \theta_{l}} u\left(y_{t+1}, \theta_{0}\right)^{\prime} \mid x_{t}\right]\right| \stackrel{p}{\rightarrow} 0
$$


for $l=1, \ldots, k$. Combining these results and $\sup _{1 \leq t \leq T}\left|\mathbb{I}_{t T}\right| \leq 1$,

$$
\left|A_{2, l}\left(\theta_{0}\right)\right| \leq\left(\sup _{t}\left|u_{T}\left(x_{t}, \theta_{0}\right)\right|\right)^{2} \sup _{t}\left|\frac{\partial V_{T}\left(x_{t}, \theta_{0}\right)^{-1}}{\partial \theta_{l}}\right| \frac{1}{2 \sqrt{T}} \sum_{t=p}^{T-1} \mathbb{I}_{t T} \stackrel{p}{\rightarrow} 0
$$

for $l=1, \ldots, k$, i.e., $\left|A_{2}\left(\theta_{0}\right)\right| \stackrel{p}{\rightarrow} 0$.

Now consider $A_{1}\left(\theta_{0}\right)$. Using again the same argument for deriving (18) with Lemma $\mathrm{A}$ for $a\left(y_{j+j}, \theta_{0}\right)=u\left(y_{j+1}, \theta_{0}\right) u\left(y_{j+1}, \theta_{0}\right)^{\prime}$ and $\frac{\partial u\left(y_{j+1}, \theta_{0}\right)}{\partial \theta^{\prime}}$, we obtain

$$
\begin{aligned}
& \sup _{t}\left|V_{T}\left(x_{t}, \theta_{0}\right)-V\left(x_{t}, \theta_{0}\right)\right|=O_{p}\left(\sqrt{\frac{\log T}{\delta_{T}^{2} T h^{p}}}\right)+O\left(\frac{h^{\omega}}{\delta_{T}}\right)=o_{p}\left(T^{-1 / 4}\right), \\
& \sup _{t}\left|\sum_{j=p}^{T-1} w_{t j} \frac{\partial u\left(y_{j+1}, \theta_{0}\right)}{\partial \theta^{\prime}}-D\left(x_{t}, \theta_{0}\right)\right|=O_{p}\left(\sqrt{\frac{\log T}{\delta_{T}^{2} T h^{p}}}\right)+O\left(\frac{h^{\omega}}{\delta_{T}}\right)=o_{p}\left(T^{-1 / 4}\right) .
\end{aligned}
$$

Thus, from (21), we have

$$
\begin{aligned}
A_{1}\left(\theta_{0}\right) & =\frac{1}{\sqrt{T}} \sum_{t=p}^{T-1} \mathbb{I}_{t T} D\left(x_{t}, \theta_{0}\right)^{\prime} V\left(x_{t}, \theta_{0}\right)^{-1} u_{T}\left(x_{t}, \theta_{0}\right)+o_{p}(1) \\
& =\frac{1}{T^{3 / 2}} \sum_{t=p}^{T-1} \mathbb{I}_{t T} \sum_{j=p}^{T-1} D\left(x_{t}, \theta_{0}\right)^{\prime} V\left(x_{t}, \theta_{0}\right)^{-1} \frac{\mathbb{K}\left(\frac{x_{j}-x_{t}}{h}\right) u\left(y_{j+1}, \theta_{0}\right)}{E\left[\mathbb{K}\left(\frac{x_{i}-x_{t}}{h}\right) \mid x_{t}\right]}+o_{p}(1),
\end{aligned}
$$

where the second equality follows from Lemma A with $a\left(y_{j+1}, \theta\right)=1$. Therefore, by applying a similar argument as Kitamura, Tripathi and Ahn (2004, pp. 1696-1698) and using the central limit theorem of $U$-statistics for absolute regular processes (Fan and $\mathrm{Li}, 1999$ ), we can show $A_{1}\left(\theta_{0}\right) \stackrel{d}{\rightarrow}$ $N\left(0, \mathcal{I}\left(\theta_{0}\right)\right)$, which implies $(19)$.

For $(20)$, note that $\frac{1}{2} \frac{\partial^{2} Q_{T}(\bar{\theta})}{\partial \theta \partial \theta^{\prime}}=\frac{1}{\sqrt{T}} \frac{\partial A_{1}(\bar{\theta})}{\partial \theta^{\prime}}+\frac{1}{\sqrt{T}} \frac{\partial A_{2}(\bar{\theta})}{\partial \theta^{\prime}}$ and

$$
\begin{aligned}
\frac{1}{\sqrt{T}} \frac{\partial A_{1}(\theta)}{\partial \theta_{l}}= & \frac{1}{T} \sum_{t=p}^{T-1} \mathbb{I}_{t T}\left[\sum_{j=p}^{T-1} w_{t j} \frac{\partial^{2} u\left(y_{j+1}, \theta\right)}{\partial \theta_{l} \partial \theta^{\prime}}\right]^{\prime} V_{T}\left(x_{t}, \theta\right)^{-1} u_{T}\left(x_{t}, \theta\right) \\
& +\frac{1}{T} \sum_{t=p}^{T-1} \mathbb{I}_{t T}\left[\sum_{j=p}^{T-1} w_{t j} \frac{\partial u\left(y_{j+1}, \theta\right)}{\partial \theta^{\prime}}\right]^{\prime} \frac{\partial V_{T}\left(x_{t}, \theta\right)^{-1}}{\partial \theta_{l}} u_{T}\left(x_{t}, \theta\right) \\
& +\frac{1}{T} \sum_{t=p}^{T-1} \mathbb{I}_{t T}\left[\sum_{j=p}^{T-1} w_{t j} \frac{\partial u\left(y_{j+1}, \theta\right)}{\partial \theta^{\prime}}\right]^{\prime} V_{T}\left(x_{t}, \theta\right)^{-1}\left[\sum_{j=p}^{T-1} w_{t j} \frac{\partial u\left(y_{j+1}, \theta\right)}{\partial \theta_{l}}\right] \\
= & A_{11}(\theta)+A_{12}(\theta)+A_{13}(\theta),
\end{aligned}
$$

for $l=1, \ldots, k$, where $A_{11}(\theta), A_{12}(\theta)$, and $A_{13}(\theta)$ are implicitly defined. Consider $A_{11}(\bar{\theta})$. From 
Lemma A with $a\left(y_{j+1}, \theta\right)=\frac{\partial^{2} u\left(y_{j+1}, \theta\right)}{\partial \theta_{l} \partial \theta^{\prime}}$ and $\bar{\theta} \stackrel{p}{\rightarrow} \theta_{0}$ (by the consistency of $\hat{\theta}$ ), we have

$$
\sup _{t}\left|\sum_{j=p}^{T-1} w_{t j} \frac{\partial^{2} u\left(y_{j+1}, \bar{\theta}\right)}{\partial \theta_{l} \partial \theta^{\prime}}\right| \leq \sup _{t} \sup _{\theta \in \mathcal{N}}\left|\sum_{j=p}^{T-1} w_{t j} \frac{\partial^{2} u\left(y_{j+1}, \theta\right)}{\partial \theta_{l} \partial \theta^{\prime}}\right|=O_{p}(1) .
$$

From (16), we obtain $\sup _{t}\left|V_{T}\left(x_{t}, \bar{\theta}\right)^{-1}\right|=O_{p}(1)$. Also, an expansion around $\bar{\theta}=\theta_{0}$ yields

$$
\begin{aligned}
\sup _{t}\left|u_{T}\left(x_{t}, \bar{\theta}\right)\right| & \leq \sup _{t}\left|u_{T}\left(x_{t}, \theta_{0}\right)\right|+\sup _{t}\left|\frac{\partial u_{T}\left(x_{t}, \tilde{\theta}\right)}{\partial \theta^{\prime}}\right|\left|\bar{\theta}-\theta_{0}\right| \\
& =o_{p}\left(T^{-1 / 4}\right)+o_{p}(1),
\end{aligned}
$$

where $\tilde{\theta}$ is a point on the line joining $\bar{\theta}$ and $\theta_{0}$, and the equality follows from $(21), \bar{\theta} \stackrel{p}{\rightarrow} \theta_{0}$, and $\sup _{t}\left|\frac{\partial u_{T}\left(x_{t}, \tilde{\theta}\right)}{\partial \theta^{\prime}}\right|=O_{p}(1)$ (by similar arguments as in the derivation of (22)). Combining these results with $\sup _{1 \leq t \leq T}\left|\mathbb{I}_{t T}\right| \leq 1$, we obtain $\left|A_{11}(\bar{\theta})\right| \stackrel{p}{\rightarrow} 0$. Similarly, we can show that $A_{12}(\bar{\theta}) \stackrel{p}{\rightarrow} 0$.

Finally consider $A_{13}(\bar{\theta})$. By an expansion around $\bar{\theta}=\theta_{0}$,

$$
\begin{aligned}
& \sup _{t}\left|\sum_{j=p}^{T-1} w_{t j} \frac{\partial u\left(y_{j+1}, \bar{\theta}\right)}{\partial \theta_{l}}-E\left[\frac{\partial u\left(y_{j+1}, \theta_{0}\right)}{\partial \theta_{l}} \mid x_{t}\right]\right| \\
& \leq \sup _{t}\left|\sum_{j=p}^{T-1} w_{t j} \frac{\partial u\left(y_{j+1}, \theta_{0}\right)}{\partial \theta_{l}}-E\left[\frac{\partial u\left(y_{j+1}, \theta_{0}\right)}{\partial \theta_{l}} \mid x_{t}\right]\right|+\sup _{t}\left|\sum_{j=p}^{T-1} w_{t j} \frac{\partial^{2} u\left(y_{j+1}, \dot{\theta}\right)}{\partial \theta_{l} \partial \theta^{\prime}}\right|\left|\bar{\theta}-\theta_{0}\right| \\
& \stackrel{p}{\rightarrow} 0
\end{aligned}
$$

where $\dot{\theta}$ is a point on the line joining $\bar{\theta}$ and $\theta_{0}$, and the convergence follows from (22), (23), and $\bar{\theta} \stackrel{p}{\rightarrow} \theta_{0}$. Similarly, we have $\sup _{t}\left|V_{T}\left(x_{t}, \bar{\theta}\right)^{-1}-V\left(x_{t}, \theta_{0}\right)\right| \stackrel{p}{\rightarrow} 0$. Combining these results, we have $A_{13}(\bar{\theta}) \stackrel{p}{\rightarrow} E\left[D\left(x_{t}, \theta_{0}\right)^{\prime} V\left(x_{t}, \theta_{0}\right) E\left[\frac{\partial u\left(y_{t+1}, \theta\right)}{\partial \theta_{l}} \mid x_{t}\right]\right]$ and thus $\frac{1}{\sqrt{T}} \frac{\partial A_{1}(\bar{\theta})}{\partial \theta^{\prime}} \stackrel{p}{\rightarrow} \mathcal{I}\left(\theta_{0}\right)$. Also, a similar but lengthy argument yields $\frac{1}{\sqrt{T}} \frac{\partial A_{2}(\bar{\theta})}{\partial \theta} \stackrel{p}{\rightarrow} 0$. Therefore, we obtain (20), which combined with the proof of (19) completes the proof of part (b) in Theorem 1.

\section{A.2 Proof of Theorem 2}

We first derive asymptotic expansions for $\hat{r}_{t}$ and $\hat{u}_{t}$. An expansion of $\hat{r}_{t}=\tilde{r}_{t} / \tilde{f}_{t}$ around $\tilde{f}_{t}=f_{t}$ yields

$$
\hat{r}_{t}=\left[\frac{1}{f_{t}}-\frac{V_{f, t}+B_{f, t}}{f_{t}^{2}}+R_{f, t}\right]\left[V_{r, t}+B_{r, t}+r_{t} f_{t}\right]
$$

for $t=1, \ldots, T-1$, where $R_{f, t}=\left(V_{f, t}+B_{f, t}\right)^{2} /\left(4 \dot{f}_{t}^{3}\right)$ and $\dot{f}_{t}$ is a point on the line joining $\tilde{f}_{t}$ and $f_{t}$. Similarly, an expansion of $\hat{u}_{t+1}=\tilde{u}_{t+1} / \tilde{f}_{t}$ around $\tilde{f}_{t}=f_{t}$ is obtained as

$$
\hat{u}_{t}=\left[\frac{1}{f_{t}}-\frac{V_{f, t}+B_{f, t}}{f_{t}^{2}}+R_{f, t}\right] \tilde{u}_{t+1}
$$


for $t=1, \ldots, T-1$. From $(24),(25)$, and an expansion of $\hat{\theta}_{L G M M 1}-\theta_{0}=\frac{T^{-1} \sum_{t=1}^{T-1} \mathbb{I}_{t T} \hat{r}_{t} \hat{u}_{t+1}}{T^{-1} \sum_{t=1}^{T-1} \mathbb{I}_{t T} \hat{r}_{t}^{2}}$ around $T^{-1} \sum_{t=1}^{T} \mathbb{I}_{t T} \hat{r}_{t}^{2}=\sigma_{r}^{2}$, we have

$$
\hat{\theta}_{L G M M 1}-\theta_{0}=M_{1}-M_{2}+M_{3}
$$

where

$$
\begin{aligned}
& M_{1}=\frac{1}{\sigma_{r}^{2}} \frac{1}{T} \sum_{t=1}^{T-1} \mathbb{I}_{t T}\left[\frac{1}{f_{t}}-\frac{V_{f, t}+B_{f, t}}{f_{t}^{2}}+R_{f, t}\right]^{2}\left[V_{r, t}+B_{r, t}+r_{t} f_{t}\right] \tilde{u}_{t+1}, \\
& M_{2}=\frac{T^{-1} \sum_{t=1}^{T} \mathbb{I}_{t T} \hat{r}_{t}^{2}-\sigma_{r}^{2}}{\sigma_{r}^{4}} \frac{1}{T} \sum_{t=1}^{T-1} \mathbb{I}_{t T}\left[\frac{1}{f_{t}}-\frac{V_{f, t}+B_{f, t}}{f_{t}^{2}}+R_{f, t}\right]^{2}\left[V_{r, t}+B_{r, t}+r_{t} f_{t}\right] \tilde{u}_{t+1}, \\
& M_{3}=R \frac{1}{T} \sum_{t=1}^{T-1} \mathbb{I}_{t T}\left[\frac{1}{f_{t}}-\frac{V_{f, t}+B_{f, t}}{f_{t}^{2}}+R_{f, t}\right]^{2}\left[V_{r, t}+B_{r, t}+r_{t} f_{t}\right] \tilde{u}_{t+1},
\end{aligned}
$$

and $R$ is the remainder term satisfying $R=O_{p}\left(\left(T^{-1} \sum_{t=1}^{T} \mathbb{I}_{t T} \hat{r}_{t}^{2}-\sigma_{r}^{2}\right)^{2}\right)$. For $M_{1}$, a lengthy calculation, combined with Assumption A4 (see Appendix A.2.1), implies

$$
\begin{aligned}
M_{1}= & \frac{1}{\sigma_{r}^{2}} \frac{1}{T} \sum_{t=1}^{T-1} \mathbb{I}_{t T} \frac{\left(V_{r, t}+B_{r, t}+r_{t} f_{t}\right) \tilde{u}_{t+1}}{f_{t}^{2}}-\frac{2}{\sigma_{r}^{2}} \frac{1}{T} \sum_{t=1}^{T-1} \mathbb{I}_{t T} \frac{\left(V_{f, t}+B_{f, t}\right) r_{t} \tilde{u}_{t+1}}{f_{t}^{2}} \\
& +O_{p}\left(a_{T}\left(a_{T}+b_{T}\right)^{2}\right) .
\end{aligned}
$$

From (24), an expansion for $T^{-1} \sum_{t=1}^{T} \mathbb{I}_{t T} \hat{r}_{t}^{2}-\sigma_{r}^{2}$ is obtained as

$$
\begin{aligned}
T^{-1} \sum_{t=1}^{T-1} \mathbb{I}_{t T} \hat{r}_{t}^{2}-\sigma_{r}^{2}= & \left(\tilde{s}_{r}^{2}-\sigma_{r}^{2}\right)+\frac{2}{T} \sum_{t=1}^{T-1} \mathbb{I}_{t T} \frac{r_{t}\left(V_{r, t}+B_{r, t}\right)}{f_{t}}-\frac{2}{T-1} \sum_{t=1}^{T-1} \mathbb{I}_{t T} \frac{r_{t}^{2}\left(V_{f, t}+B_{f, t}\right)}{f_{t}}(27) \\
& +O_{p}\left(\left(a_{T}+b_{T}\right)^{2}\right)
\end{aligned}
$$

From (27) and a similar argument as in the derivation of (26), an expansion for $M_{2}$ is given by

$$
\begin{aligned}
M_{2}= & \frac{1}{\sigma_{r}^{4}}\left(\left(\tilde{s}_{r}^{2}-\sigma_{r}^{2}\right)+\frac{2}{T} \sum_{t=1}^{T-1} \mathbb{I}_{t T} \frac{r_{t}\left(V_{r, t}+B_{r, t}\right)}{f_{t}}-\frac{2}{T} \sum_{t=1}^{T-1} \mathbb{I}_{t T} \frac{r_{t}^{2}\left(V_{f, t}+B_{f, t}\right)}{f_{t}}\right) \frac{1}{T} \sum_{t=1}^{T-1} \mathbb{I}_{t T} \frac{r_{t} \tilde{u}_{t+1}}{f_{t}} \\
& +O_{p}\left(a_{T}\left(a_{T}+b_{T}\right)^{2}\right) .
\end{aligned}
$$

Also, under our assumptions, $M_{3}$ satisfies

$$
M_{3}=O_{p}\left(a_{T}\left(a_{T}+b_{T}\right)^{2}\right)
$$

Combining these results, we obtain the conclusion. 


\section{A.2.1 Derivation of (26)}

A direct calculation yields

$$
\begin{aligned}
M_{1}= & \frac{1}{\sigma_{r}^{2}} \frac{1}{T-1} \sum_{t=1}^{T-1} \mathbb{I}_{t T} \frac{1}{f_{t}^{2}}\left[V_{r, t}+B_{r, t}+r_{t} f_{t}\right] \tilde{u}_{t+1} \\
& +\frac{1}{\sigma_{r}^{2}} \frac{1}{T-1} \sum_{t=1}^{T-1} \mathbb{I}_{t T} \frac{\left(V_{f, t}+B_{f, t}\right)^{2}}{f_{t}^{4}}\left[V_{r, t}+B_{r, t}+r_{t} f_{t}\right] \tilde{u}_{t+1} \\
& +\frac{1}{\sigma_{r}^{2}} \frac{1}{T-1} \sum_{t=1}^{T-1} \mathbb{I}_{t T} R_{f, t}^{2}\left[V_{r, t}+B_{r, t}+r_{t} f_{t}\right] \tilde{u}_{t+1} \\
& -\frac{2}{\sigma_{r}^{2}} \frac{1}{T-1} \sum_{t=1}^{T-1} \mathbb{I}_{t T} \frac{V_{f, t}+B_{f, t}}{f_{t}^{3}}\left[V_{r, t}+B_{r, t}+r_{t} f_{t}\right] \tilde{u}_{t+1} \\
& +\frac{2}{\sigma_{r}^{2}} \frac{1}{T-1} \sum_{t=1}^{T-1} \mathbb{I}_{t T} \frac{R_{f, t}}{f_{t}}\left[V_{r, t}+B_{r, t}+r_{t} f_{t}\right] \tilde{u}_{t+1} \\
& -\frac{2}{\sigma_{r}^{2}} \frac{1}{T-1} \sum_{t=1}^{T-1} \mathbb{I}_{t T} \frac{\left(V_{f, t}+B_{f, t}\right) R_{f, t}}{f_{t}^{2}}\left[V_{r, t}+B_{r, t}+r_{t} f_{t}\right] \tilde{u}_{t+1} \\
= & M_{11}+M_{12}+M_{13}-2 M_{14}+2 M_{15}-2 M_{16} .
\end{aligned}
$$

$M_{11}$ is the main term. For $M_{12}$, our assumptions and the law of large numbers $\left(\frac{1}{T-1} \sum_{t=1}^{T-1} \mathbb{I}_{t T}\left|\frac{1}{f_{t}^{4}}\right|=\right.$ $O_{p}(1)$ and $\left.\frac{1}{T-1} \sum_{t=1}^{T-1} \mathbb{I}_{t T}\left|\frac{r_{t}}{f_{t}^{3}}\right|=O_{p}(1)\right)$ imply

$$
\begin{aligned}
\left|M_{12}\right| \leq & \frac{1}{\sigma_{r}^{2}}\left(\sup _{t}\left|V_{f, t}+B_{f, t}\right|\right)^{2} \sup _{t}\left|\tilde{u}_{t+1}\right| \\
& \times\left\{\sup _{t}\left|V_{r, t}+B_{r, t}\right| \frac{1}{T-1} \sum_{t=1}^{T-1} \mathbb{I}_{t T}\left|\frac{1}{f_{t}^{4}}\right|+\frac{1}{T-1} \sum_{t=1}^{T-1} \mathbb{I}_{t T}\left|\frac{r_{t}}{f_{t}^{3}}\right|\right\} \\
= & O_{p}\left(a_{T}\left(a_{T}+b_{T}\right)^{2}\right) .
\end{aligned}
$$

For $M_{13}$, we have

$$
\begin{aligned}
\left|M_{13}\right| & \leq \frac{1}{\sigma_{r}^{2}} \sup _{t}\left|\tilde{u}_{t+1}\right|\left(\sup _{t}\left|V_{f, t}+B_{f, t}\right|\right)^{2}\left\{\sup _{t}\left|V_{r, t}+B_{r, t}\right| \frac{1}{T-1} \sum_{t=1}^{T-1} \mathbb{I}_{t T}\left|\frac{1}{4 \dot{f}_{t}^{3}}\right|+\frac{1}{T-1} \sum_{t=1}^{T-1} \mathbb{I}_{t T}\left|\frac{r_{t} f_{t}}{4 \dot{f}_{t}^{3}}\right|\right\} \\
& =O_{p}\left(a_{T}\left(a_{T}+b_{T}\right)^{2}\right)
\end{aligned}
$$

where the equality follows from Assumption A4, $\sup _{t}\left|\frac{\hat{f}_{t}}{f_{t}}-1\right| \stackrel{p}{\rightarrow} 0$, and the law of large numbers. For $M_{14}$, we have

$$
M_{14}=\frac{1}{\sigma_{r}^{2}} \frac{1}{T-1} \sum_{t=1}^{T-1} \mathbb{I}_{t T} \frac{V_{f, t}+B_{f, t}}{f_{t}^{2}} r_{t} \tilde{u}_{t+1}+O_{p}\left(a_{T}\left(a_{T}+b_{T}\right)^{2}\right)
$$


where the first equality follows from

$$
\begin{aligned}
& \left|\frac{1}{\sigma_{r}^{2}} \frac{1}{T-1} \sum_{t=1}^{T-1} \mathbb{I}_{t T} \frac{V_{f, t}+B_{f, t}}{f_{t}^{3}}\left[V_{r, t}+B_{r, t}\right] \tilde{u}_{t+1}\right| \\
\leq & \frac{1}{\sigma_{r}^{2}} \sup _{t}\left|V_{f, t}+B_{f, t}\right| \sup _{t}\left|V_{r, t}+B_{r, t}\right| \sup _{t}\left|\tilde{u}_{t+1}\right| \times\left\{\frac{1}{T-1} \sum_{t=1}^{T-1} \mathbb{I}_{t T}\left|\frac{1}{f_{t}^{3}}\right|\right\}=O_{p}\left(a_{T}\left(a_{T}+b_{T}\right)^{2}\right) .
\end{aligned}
$$

For $M_{15}$,

$$
\begin{aligned}
\left|M_{15}\right| & \leq \frac{1}{\sigma_{r}^{2}} \sup _{t}\left|\tilde{u}_{t+1}\right|\left(\sup _{t}\left|V_{f, t}+B_{f, t}\right|\right)^{2}\left\{\sup _{t}\left|V_{r, t}+B_{r, t}\right| \frac{1}{T-1} \sum_{t=1}^{T-1} \mathbb{I}_{t T}\left|\frac{1}{4 f_{t} \dot{f}_{t}^{3}}\right|+\frac{1}{T-1} \sum_{t=1}^{T-1} \mathbb{I}_{t T}\left|\frac{r_{t}}{4 \dot{f}_{t}^{3}}\right|\right\} \\
& =O_{p}\left(a_{T}\left(a_{T}+b_{T}\right)^{2}\right) .
\end{aligned}
$$

Similarly, for $M_{16}$,

$$
\begin{aligned}
\left|M_{16}\right| & \leq \frac{1}{\sigma_{r}^{2}} \sup _{t}\left|\tilde{u}_{t+1}\right|\left(\sup _{t}\left|V_{f, t}+B_{f, t}\right|\right)^{3}\left\{\sup _{t}\left|V_{r, t}+B_{r, t}\right| \frac{1}{T-1} \sum_{t=1}^{T-1} \mathbb{I}_{t T}\left|\frac{1}{4 f_{t}^{2} \dot{f}_{t}^{3}}\right|+\frac{1}{T-1} \sum_{t=1}^{T-1} \mathbb{I}_{t T}\left|\frac{r_{t}}{4 f_{t} \dot{f}_{t}^{3}}\right|\right\} \\
& =O_{p}\left(a_{T}\left(a_{T}+b_{T}\right)^{3}\right) .
\end{aligned}
$$

Combining these results, we obtain (26).

\section{A.3 Proof of Theorem 3}

Note that

$$
\begin{aligned}
E\left[A_{L G M M}^{*}\right] & =\frac{1}{\sigma_{r}^{2}} \frac{1}{T^{2}} \sum_{t=1}^{T-1} \sum_{j=1}^{T-1} E\left[\frac{r_{t}}{f_{t}} \frac{1}{h} \mathbb{K}\left(\frac{r_{t}-r_{j}}{h}\right) u_{j+1}\right] \\
& =\frac{1}{\sigma_{r}^{2}} \frac{1}{T^{2}} \sum_{t=1}^{T-1} \sum_{j=1}^{t-1} E\left[\frac{r_{t}}{f_{t}} \frac{1}{h} \mathbb{K}\left(\frac{r_{t}-r_{j}}{h}\right) u_{j+1}\right] \\
& =\frac{1}{\sigma_{r}^{2}} \frac{1}{T^{2}} \sum_{t=1}^{T-1} \sum_{j=1}^{t-1} A_{t, j}
\end{aligned}
$$


where the second equality follows from the law of iterated expectation. Let $f_{u}$ be the density function of $u_{t}$. For $A_{t, t-1}$, we have

$$
\begin{aligned}
A_{t, t-1} & =E\left[\frac{r_{t}}{f\left(r_{t}\right)} \frac{1}{h} \mathbb{K}\left(\frac{r_{t}-r_{t-1}}{h}\right) u_{t}\right] \\
& =E\left[\frac{\theta_{0} r_{t-1}+u_{t}}{f\left(\theta_{0} r_{t-1}+u_{t}\right)} \frac{1}{h} \mathbb{K}\left(\frac{\left(\theta_{0}-1\right) r_{t-1}+u_{t}}{h}\right) u_{t}\right] \\
& =E\left[\int \frac{a h+r_{t-1}}{f\left(a h+r_{t-1}\right)} \mathbb{K}(a)\left(a h+\left(1-\theta_{0}\right) r_{t-1}\right) f_{u}\left(a h+\left(1-\theta_{0}\right) r_{t-1}\right) d a\right] \\
& =E\left[\frac{\left(1-\theta_{0}\right) r_{t-1}^{2} f_{u}\left(\left(1-\theta_{0}\right) r_{t-1}\right)}{f\left(r_{t-1}\right)}\right]+O\left(h^{\omega}\right) \\
& =\int \frac{b^{2}}{\left(1-\theta_{0}\right)^{2}} f_{u}(b) d b+O\left(h^{\omega}\right) \\
& =\frac{1}{\left(1-\theta_{0}\right)^{2}}+O\left(h^{\omega}\right),
\end{aligned}
$$

where the third equality follows from the law of iterated expectations and a change of variables $\left(a=\frac{\left(\theta_{0}-1\right) r_{t-1}+u_{t}}{h}\right)$, the fourth equality follows from an expansion around $a=0$ and the property of the $\omega$-th order kernel $\mathbb{K}$, and the fifth equality follows from a change of variables $\left(b=\left(1-\theta_{0}\right) r_{t-1}\right)$. Similarly, for $A_{t, t-l}(l=2, \ldots, t-1)$,

$$
\begin{aligned}
A_{t, t-l} & =E\left[\frac{r_{t}}{f\left(r_{t}\right)} \frac{1}{h} \mathbb{K}\left(\frac{r_{t}-r_{t-l}}{h}\right) u_{t-l+1}\right] \\
& =E\left[\begin{array}{c}
\frac{\theta_{0}^{l} r_{t-l}+u_{t}+\theta_{0} u_{t-1}+\cdots+\theta_{0}^{l-1} u_{t-l+1}}{f\left(\theta_{0}^{l} r_{t-l}+u_{t}+\theta_{0} u_{t-1}+\cdots+\theta_{0}^{l-1} u_{t-l+1}\right)} \\
\times \frac{1}{h} \mathbb{K}\left(\frac{\left(\theta_{0}^{l}-1\right) r_{t-l}+u_{t}+\theta_{0} u_{t-1}+\cdots+\theta_{0}^{l-1} u_{t-l+1}}{h}\right) u_{t-l+1}
\end{array}\right] \\
& =E\left[\int \frac{a h+r_{t-l}}{f\left(a h+r_{t-l}\right)} \mathbb{K}(a) u_{t-l+1} f_{u}\left(a h+\left(1-\theta_{0}^{l}\right) r_{t-l}-\theta_{0} u_{t-1}-\cdots-\theta_{0}^{l-1} u_{t-l+1}\right) d a\right] \\
& =E\left[\frac{r_{t-l}}{f\left(r_{t-l}\right)} u_{t-l+1} f_{u}\left(\left(1-\theta_{0}^{l}\right) r_{t-l}-\theta_{0} u_{t-1}-\cdots-\theta_{0}^{l-1} u_{t-l+1}\right)\right]+O\left(h^{\omega}\right) \\
& =E\left[\left(\int r f_{u}\left(\left(1-\theta_{0}^{l}\right) r-\theta_{0} u_{t-1}-\cdots-\theta_{0}^{l-1} u_{t-l+1}\right) d r\right) u_{t-l+1}\right]+O\left(h^{\omega}\right) \\
& =E\left[\left(\int \frac{\left.\left.b+\theta_{0} u_{t-1}+\cdots+\theta_{0}^{l-1} u_{t-l+1} f_{u}(b) d b\right) u_{t-l+1}\right]+O\left(h^{\omega}\right)}{\left(1-\theta_{0}^{l}\right)^{2}}\right.\right. \\
& =\frac{1}{\left(1-\theta_{0}^{l}\right)^{2}} E\left[\left(\theta_{0} u_{t-1}+\cdots+\theta_{0}^{l-1} u_{t-l+1}\right) u_{t-l+1}\right]+O\left(h^{\omega}\right) \\
& =\frac{\theta_{0}^{l-1}}{\left(1-\theta_{0}^{l}\right)^{2}}+O\left(h^{\omega}\right),
\end{aligned}
$$

where the second equality follows from model (7), the third equality follows from the law of iterated expectations (with respect to $u_{t}$ given the information at time $t-1$ ) and a change of variables 
( $\left.a=\frac{\left(\theta_{0}^{l}-1\right) r_{t-l}+u_{t}+\theta_{0} u_{t-1}+\cdots+\theta_{0}^{l-1} u_{t-l+1}}{h}\right)$, the fourth equality follows from an expansion around $a=0$ and the property of the $\omega$-th order kernel $\mathbb{K}$, the fifth equality follows from the law of iterated expectations (with respect to $r_{t-l}$ given $\left.\left(u_{t-1}, \ldots, u_{t-l+1}\right)\right)$ and independence between $r_{t-l}$ and $\left(u_{t-1}, \ldots, u_{t-l+1}\right)$, and the sixth equality follows from a change of variable $\left(b=\left(1-\theta_{0}^{l}\right) r-\theta_{0} u_{t-1}-\right.$ $\left.\cdots-\theta_{0}^{l-1} u_{t-l+1}\right)$. Combining these results with $\sigma_{r}^{-2}=1-\theta_{0}^{2}$, we obtain the conclusion. 


\section{References}

[1] Aït-Sahalia, Y., 1996. Testing continuous time models of the spot interest rate. Review of Financial Studies 9, 385-426.

[2] Anatolyev, S., 2005. GMM, GEL, serial correlation, and asymptotic bias. Econometrica 73, 983-1002.

[3] Antoine, B., Bonnal, H., Renault, E., 2007. On the efficient use of the informational content of estimating equations: Implied probabilities and Euclidean empirical likelihood. Journal of Econometrics 138, 461-487.

[4] Ball, C.A., Torous, W.N., 1996. Unit roots and the estimation of interest rate dynamics. Journal of Empirical Finance 3, 215-238.

[5] Beach, C.M., MacKinnon, J.G., 1978. A maximum likelihood procedure for regression with autocorrelated errors. Econometrica 46, 51-58.

[6] Borkovec, M., 2001, Asymptotic behaviour of the sample autocovariance and autocorrelation function of the $\mathrm{AR}(1)$ process with $\mathrm{ARCH}(1)$ errors. Bernoulli 7, 847-872.

[7] Borkovec, M., Klüppelberg, C., 2001. The tail of the stationary distribution of an autoregressive process with ARCH(1) errors. Annals of Applied Probability 11, 1220-1241.

[8] Carrasco, M., Chen, X., 2002. Mixing and moment properties of various GARCH and stochastic volatility models. Econometric Theory 18, 17-39.

[9] Carrasco, M., Chernov, M., Florens, J.-P., Ghysels, E., 2007. Efficient estimation of general dynamic models with a continuum of moment conditions. Journal of Econometrics 140, 529573.

[10] Carrasco, M., Florens, J.P., 2000. Generalization of GMM to a continuum of moment conditions. Econometric Theory 16, 797-834.

[11] Carrasco, M., Florens, J.P., 2004. On the asymptotic efficiency of GMM. Manuscript, University of Rochester.

[12] Chamberlain, G., 1987. Asymptotic efficiency in estimation with conditional moment restrictions. Journal of Econometrics 34, 395-334.

[13] Chapman, D., Pearson, N., 2000. Is the short rate drift actually nonlinear? Journal of Finance $55,355-388$.

[14] Chen, S.X., Härdle, W., Li, M., 2003. An empirical likelihood goodness-of-fit test for time series. Journal of the Royal Statistical Society B 65, 663-678.

[15] Cox, J.C., Ingersoll, J.E.Jr., Ross, S.A., 1985. A theory of the term structure of interest rates. Econometrica 53, 385-408.

[16] Davidson, J., 2000. Econometric Theory. Oxford: Blackwell Publishers. 
[17] Dominguez, M., Lobato, I., 2004. Consistent estimation of models defined by conditional moment restrictions. Econometrica 72, 1601-1615.

[18] Donald, S.G., Imbens, G.W., Newey, W.K., 2003. Empirical likelihood estimation and consistent tests with conditional moment restrictions. Journal of Econometrics 117, 55-93.

[19] Donald, S.G., Newey, W.K., 2000. A jackknife interpretation of the continuous updating estimator. Economics Letters 67, 239-243.

[20] Fan, Y., Li, Q., 1999. Central limit theorem for degenerate U-statistics of absolutely regular processes with applications to model specification testing. Journal of Nonparametric Statistics $10,245.271$.

[21] Gagliardini, P., Gourieroux, C., Renault, E., 2007. Efficient derivative pricing by extended method of moments. Manuscript, University of Lugano.

[22] Giersbergen, N.P.A.v., 2005. On the effect of deterministic terms on the bias in stable AR models. Economics Letters 89, 75-82.

[23] Guégan, D., Diebolt, J., 1994. Probabilistic properties of the $\beta$-ARCH model. Statistica Sinica $4,71-87$.

[24] Hansen, L.P., Heaton, J., Yaron, A., 1996. Finite-sample properties of some alternative GMM estimators. Journal of Business and Economic Statistics 14, 262-280.

[25] Kendall, M.G., 1954. Note on bias in the estimation of autocorrelation. Biometrika 41, 403-404.

[26] Kitamura, Y., Tripathi, G., Ahn, H., 2004. Empirical likelihood-based inference in conditional moment restriction models. Econometrica 72, 1667-1714.

[27] Kristensen, D., 2009. Uniform convergence rates of kernel estimators with heterogeneous dependent data. Econometric Theory 25, 1433-1445.

[28] Kuersteiner, G., 2001. Optimal instrumental variables estimation fro ARMA models. Journal of Econometrics 104, 359-405.

[29] Kuersteiner, G., 2002. Efficient estimation for autoregressive models with conditional heteroskedasticity. Econometric Theory 18, 547-583.

[30] Kuersteiner, G., 2006. Moment selection and bias reduction for GMM in conditionally heteroskedastic models. In: D. Corbea, S. Durlauf and B.E. Hansen, eds, Econometric Theory and Practice: Frontiers of Analysis and Applied Research: Essays in Honor of Peter C.B. Phillips, Cambridge: Cambridge University Press.

[31] Lavergne, P., Patilea, V., 2008. Efficient smooth GMM and dimension reduction. Manuscript, Simon Fraser University.

[32] Lewbel, A., 2007. A local generalized method of moments estimator. Economics Letters 94, 124-128.

[33] Ling, S., 2004. Estimation and testing stationarity for double-autoregressive models. Journal of the Royal Statistical Society B 66, 63-78. 
[34] Maercker, G., 1995. Efficient estimation in AR models with ARCH errors. Technical report, Institut für Mathematische Stochastik, TU Braunschweig.

[35] Mariott, F.H.C., Pope, J.A., 1954. Bias in the estimation of autocorrelations. Biometrika 41, 390-402.

[36] Masry, E., Tjøstheim, D., 1995. Nonparametric estimation and identification of nonlinear arch time series. Econometric Theory 11, 258-289.

[37] Meyn, S.P., Tweedie, R.L., 1993. Markov Chains and Stochastic Stability. Berlin: SpringerVerlag.

[38] Newey, W.K., 1990. Efficient instrumental variables estimation of nonlinear models. Econometrica 58, 809-837.

[39] Newey, W.K., 1993. Efficient estimation of models with conditional moment restrictions. In: G.S. Maddala, C.R. Rao and H.D. Vinod, eds., Handbook of Statistics, Vol. 11 (Elsevier, Amsterdam) 419-454.

[40] Newey, W.K., McFadden, D.L., 1994. Large sample estimation and hypothesis testing. In: R.F. Engle and D.L. McFadden, eds., Handbook of Econometrics, Vol. 4 (Elsevier, Amsterdam) 2111-2245.

[41] Newey, W.K., Smith, R.J., 2004. Higher order properties of GMM and generalized empirical likelihood estimators. Econometrica 72, 219-255.

[42] Phillips, P.C.B., Yu, J., 2005. Jackknifing bond option prices. Review of Financial Studies 18, 707-742.

[43] Smith, R.J., 2007. Efficient information theoretic inference for conditional moment restrictions. Journal of Econometrics 138, 430-460.

[44] Su, L., White, H., 2003. Testing conditional independence via empirical likelihood. UCSD working paper 2003-14.

[45] West, K.D., Wong, K., Anatolyev, S., 2009. Instrumental variables estimation of heteroskedastic linear models using all lags of instruments. Econometric Reviews 28, 441-467. 
TABLE 1. Finite-sample properties of OLS, GLS, optimal instrumental variables (OIV), CarrascoFlorens $(\mathrm{CF})$ and LGMM estimators of the slope parameter in an $\mathrm{AR}(1)$ model $(T=100)$.

\begin{tabular}{l|cccc|cccc|cccc}
\hline \hline & \multicolumn{5}{|c|}{$\theta_{0}=0.4$} & \multicolumn{5}{c|}{$\theta_{0}=0.7$} & \multicolumn{5}{c}{$\theta_{0}=0.95$} \\
\hline & mean & med & sd & rmse & mean & med & sd & rmse & mean & med & sd & rmse \\
\hline$\beta_{0}=0$ & & & & & & & & & & & \\
\hline OLS & -0.022 & -0.019 & 0.093 & 0.095 & -0.032 & -0.025 & 0.076 & 0.083 & -0.044 & -0.034 & 0.050 & 0.066 \\
GLS & -0.022 & -0.019 & 0.093 & 0.095 & -0.032 & -0.025 & 0.076 & 0.083 & -0.044 & -0.034 & 0.050 & 0.066 \\
OIV & -0.022 & -0.018 & 0.094 & 0.096 & -0.032 & -0.025 & 0.078 & 0.084 & -0.048 & -0.038 & 0.051 & 0.070 \\
CF & -0.020 & -0.016 & 0.094 & 0.096 & -0.027 & -0.020 & 0.078 & 0.082 & -0.039 & -0.029 & 0.050 & 0.063 \\
LGMM & -0.018 & -0.015 & 0.101 & 0.103 & -0.021 & -0.014 & 0.083 & 0.086 & -0.029 & -0.019 & 0.051 & 0.058 \\
\hline$\beta_{0}=0.5$ & & & & & & & & & & & & \\
\hline OLS & -0.029 & -0.024 & 0.133 & 0.136 & -0.041 & -0.030 & 0.101 & 0.109 & -0.047 & -0.036 & 0.057 & 0.074 \\
GLS & -0.023 & -0.019 & 0.109 & 0.112 & -0.031 & -0.023 & 0.081 & 0.087 & -0.037 & -0.027 & 0.047 & 0.060 \\
OIV & -0.037 & -0.030 & 0.131 & 0.136 & -0.048 & -0.037 & 0.100 & 0.110 & -0.053 & -0.042 & 0.060 & 0.080 \\
CF & -0.017 & -0.012 & 0.124 & 0.125 & -0.027 & -0.018 & 0.095 & 0.099 & -0.039 & -0.027 & 0.054 & 0.066 \\
LGMM & -0.006 & -0.002 & 0.126 & 0.127 & -0.009 & 0.002 & 0.098 & 0.099 & -0.025 & -0.014 & 0.052 & 0.058 \\
\hline$\beta_{0}=0.9$ & & & & & & & & & & & & \\
\hline OLS & -0.040 & -0.029 & 0.181 & 0.185 & -0.056 & -0.036 & 0.139 & 0.150 & -0.056 & -0.038 & 0.076 & 0.094 \\
GLS & -0.021 & -0.017 & 0.113 & 0.115 & -0.025 & -0.018 & 0.078 & 0.082 & -0.025 & -0.017 & 0.040 & 0.047 \\
OIV & -0.057 & -0.044 & 0.177 & 0.186 & -0.074 & -0.053 & 0.140 & 0.158 & -0.060 & -0.045 & 0.081 & 0.101 \\
CF & -0.018 & -0.008 & 0.165 & 0.166 & -0.032 & -0.017 & 0.125 & 0.129 & -0.042 & -0.027 & 0.065 & 0.077 \\
LGMM & 0.005 & 0.008 & 0.158 & 0.158 & 0.004 & 0.015 & 0.119 & 0.119 & -0.019 & -0.007 & 0.055 & 0.058 \\
\hline
\end{tabular}

Notes: The statistics in the table are computed from 10,000 samples generated from the $\operatorname{AR}(1)$ process $r_{t+1}=\theta_{0} r_{t}+u_{t+1}$ with $T=100, \theta_{0}=0.4,0.7,0.95$, and $u_{t+1}=\sigma_{t+1} \varepsilon_{t+1}$, where $\varepsilon_{t+1} \sim$ $\operatorname{iidN}(0,1)$ and $\sigma_{t+1}=\sqrt{\left(1-\beta_{0}\right)+\beta_{0} u_{t}^{2}}$ for $\beta_{0}=0,0.5,0.9$. The estimated model includes an intercept. "mean", "med", "sd" and "rmse" denote the mean bias, median bias, standard deviation and root mean square error, respectively. 
TABLE 2. Finite-sample properties of OLS, GLS, optimal instrumental variables (OIV), CarrascoFlorens $(\mathrm{CF})$ and LGMM estimators of the slope parameter in an $\mathrm{AR}(1)$ model $(T=500)$.

\begin{tabular}{l|cccc|cccc|cccc}
\hline \hline & \multicolumn{5}{|c|}{$\theta_{0}=0.4$} & \multicolumn{5}{c|}{$\theta_{0}=0.7$} & \multicolumn{5}{c}{$\theta_{0}=0.95$} \\
\hline & mean & med & sd & rmse & mean & med & sd & rmse & mean & med & sd & rmse \\
\hline$\beta_{0}=0$ & & & & & & & & & & & & \\
\hline OLS & -0.005 & -0.004 & 0.041 & 0.041 & -0.006 & -0.005 & 0.032 & 0.033 & -0.008 & -0.006 & 0.016 & 0.018 \\
GLS & -0.005 & -0.004 & 0.041 & 0.041 & -0.006 & -0.005 & 0.032 & 0.033 & -0.008 & -0.006 & 0.016 & 0.018 \\
OIV & -0.005 & -0.005 & 0.041 & 0.041 & -0.006 & -0.006 & 0.032 & 0.033 & -0.008 & -0.006 & 0.016 & 0.018 \\
CF & -0.004 & -0.004 & 0.042 & 0.042 & -0.005 & -0.004 & 0.033 & 0.033 & -0.006 & -0.004 & 0.016 & 0.017 \\
LGMM & -0.004 & -0.004 & 0.042 & 0.042 & -0.004 & -0.003 & 0.033 & 0.034 & -0.004 & -0.002 & 0.016 & 0.017 \\
\hline$\beta_{0}=0.5$ & & & & & & & & & & & & \\
\hline OLS & -0.008 & -0.007 & 0.068 & 0.068 & -0.010 & -0.008 & 0.048 & 0.049 & -0.009 & -0.007 & 0.019 & 0.021 \\
GLS & -0.005 & -0.004 & 0.049 & 0.049 & -0.006 & -0.005 & 0.034 & 0.035 & -0.007 & -0.005 & 0.014 & 0.016 \\
OIV & -0.010 & -0.010 & 0.066 & 0.067 & -0.011 & -0.009 & 0.044 & 0.046 & -0.009 & -0.007 & 0.018 & 0.020 \\
CF & -0.003 & -0.002 & 0.055 & 0.055 & -0.004 & -0.003 & 0.041 & 0.041 & -0.005 & -0.003 & 0.018 & 0.018 \\
LGMM & -0.001 & -0.001 & 0.053 & 0.053 & -0.001 & 0.001 & 0.041 & 0.041 & -0.002 & 0.001 & 0.017 & 0.017 \\
\hline$\beta_{0}=0.9$ & & & & & & & & & & & & \\
\hline OLS & -0.019 & -0.014 & 0.130 & 0.132 & -0.025 & -0.016 & 0.095 & 0.098 & -0.016 & -0.009 & 0.035 & 0.039 \\
GLS & -0.004 & -0.004 & 0.050 & 0.050 & -0.005 & -0.003 & 0.031 & 0.031 & -0.004 & -0.003 & 0.011 & 0.012 \\
OIV & -0.030 & -0.023 & 0.125 & 0.129 & -0.033 & -0.021 & 0.088 & 0.094 & -0.012 & -0.008 & 0.031 & 0.033 \\
CF & -0.001 & 0.001 & 0.086 & 0.086 & -0.003 & 0.000 & 0.063 & 0.063 & -0.004 & -0.001 & 0.023 & 0.023 \\
LGMM & 0.002 & 0.002 & 0.064 & 0.064 & 0.004 & 0.005 & 0.050 & 0.051 & 0.002 & 0.004 & 0.018 & 0.018 \\
\hline
\end{tabular}

Notes: The statistics in the table are computed from 10,000 samples generated from the $\operatorname{AR}(1)$ process $r_{t+1}=\theta_{0} r_{t}+u_{t+1}$ with $T=500, \theta_{0}=0.4,0.7,0.95$, and $u_{t+1}=\sigma_{t+1} \varepsilon_{t+1}$, where $\varepsilon_{t+1} \sim$ $\operatorname{iidN}(0,1)$ and $\sigma_{t+1}=\sqrt{\left(1-\beta_{0}\right)+\beta_{0} u_{t}^{2}}$ for $\beta_{0}=0,0.5,0.9$. The estimated model includes an intercept. "mean", "med", "sd" and "rmse" denote the mean bias, median bias, standard deviation and root mean square error, respectively. 
TABLE 3. Bias properties of OLS and LGMM estimators of the slope parameter in a zero-mean AR(1) model with iid errors.

\begin{tabular}{lccccc}
\hline \hline & mean bias & sd & rmse & $E[A]$ & $E[B]$ \\
\hline$T=50$ & & & & & \\
\hline OLS & -0.0309 & 0.0646 & 0.0716 & 0.0002 & 0.0303 \\
LGMM & -0.0128 & 0.0621 & 0.0634 & 0.0108 & 0.0310 \\
\hline$T=100$ & & & & & \\
\hline OLS & -0.0167 & 0.0395 & 0.0429 & 0.0000 & 0.0164 \\
LGMM & -0.0040 & 0.0383 & 0.0385 & 0.0082 & 0.0168 \\
\hline$T=200$ & & & & & \\
\hline OLS & -0.0093 & 0.0260 & 0.0276 & -0.0002 & 0.0090 \\
LGMM & -0.0010 & 0.0258 & 0.0259 & 0.0055 & 0.0090 \\
\hline$T=400$ & & & & & \\
\hline OLS & -0.0048 & 0.0172 & 0.0178 & 0.0000 & 0.0048 \\
LGMM & 0.0000 & 0.0173 & 0.0173 & 0.0034 & 0.0047 \\
\hline$T=800$ & & & & & \\
\hline OLS & -0.0026 & 0.0118 & 0.0121 & -0.0001 & 0.0024 \\
LGMM & 0.0001 & 0.0119 & 0.0119 & 0.0018 & 0.0024 \\
\hline$T=1600$ & & & & & \\
\hline OLS & -0.0011 & 0.0080 & 0.0081 & 0.0000 & 0.0012 \\
LGMM & 0.0002 & 0.0081 & 0.0081 & 0.0011 & 0.0012 \\
\hline$T=3200$ & & & & & \\
\hline OLS & -0.0006 & 0.0055 & 0.0055 & 0.0000 & 0.0006 \\
LGMM & 0.0001 & 0.0055 & 0.0055 & 0.0005 & 0.0006 \\
\hline
\end{tabular}

Notes: The statistics in the table are computed from 5,000 samples generated from the $\operatorname{AR}(1)$ process $r_{t+1}=\theta_{0} r_{t}+u_{t+1}$ with $\theta_{0}=0.95$ and $u_{t+1} \sim \operatorname{iidN}(0,1)$. "sd" and "rmse" denote the standard deviation and root mean square error, respectively. The last two columns of the table are Monte Carlo averages of the terms $E\left[A_{O L S}\right]$ and $E\left[B_{O L S}\right]$ for the OLS estimator and $E\left[A_{L G M M}\right]$ and $E\left[B_{L G M M}\right]$ for the LGMM estimator which are defined in Section 4.1. 
TABLE 4. Finite-sample properties of GLS, Carrasco-Florens (CF) and LGMM estimators of the parameters of the CIR model and bond and option prices.

\begin{tabular}{lcccccc}
\hline \hline & intercept & $\kappa$ & $\mu$ & $\sigma$ & bond & option \\
\hline true & 0.0184 & 0.2146 & 0.0857 & 0.0783 & 83.763 & 1.931 \\
\hline GLS & & & & & & \\
median & 0.0488 & 0.5938 & 0.0825 & 0.0852 & 82.030 & 0.756 \\
mean & 0.0587 & 0.6931 & 0.0866 & 0.0926 & 81.613 & 1.127 \\
sd & 0.0455 & 0.4538 & 0.0279 & 0.0222 & 3.262 & 1.190 \\
rmse & 0.0608 & 0.6595 & 0.0279 & 0.0264 & 3.907 & 1.436 \\
\hline CF & & & & & & \\
median & 0.0497 & 0.6011 & 0.0826 & 0.0852 & 82.000 & 0.711 \\
mean & 0.0586 & 0.6950 & 0.0864 & 0.0926 & 81.587 & 1.110 \\
sd & 0.0407 & 0.4281 & 0.0273 & 0.0222 & 3.241 & 1.191 \\
rmse & 0.0572 & 0.6435 & 0.0273 & 0.0264 & 3.904 & 1.446 \\
\hline LGMM & & & & & & \\
median & 0.0328 & 0.4030 & 0.0833 & 0.0852 & 83.022 & 1.458 \\
mean & 0.0403 & 0.4805 & 0.0873 & 0.0926 & 82.602 & 1.652 \\
sd & 0.0324 & 0.3638 & 0.0297 & 0.0223 & 2.921 & 1.384 \\
rmse & 0.0391 & 0.4506 & 0.0298 & 0.0265 & 3.143 & 1.412 \\
\hline
\end{tabular}

Notes: The statistics in the table are computed from 5,000 samples generated from the CIR model with $\triangle=1 / 52$ and $T=600$. "sd" and "rmse" denote the standard deviation and root mean square error, respectively. The prices of a three-year zero-coupon discount bond and a one-year European call option on a three-year bond with face value of $\$ 100$ and a strike price of $\$ 87$ are computed analytically as in Cox, Ingersoll and Ross (1985) with an initial interest rate of $5 \%$. The first row "true" reports the true values of the parameters, bond and option prices. 

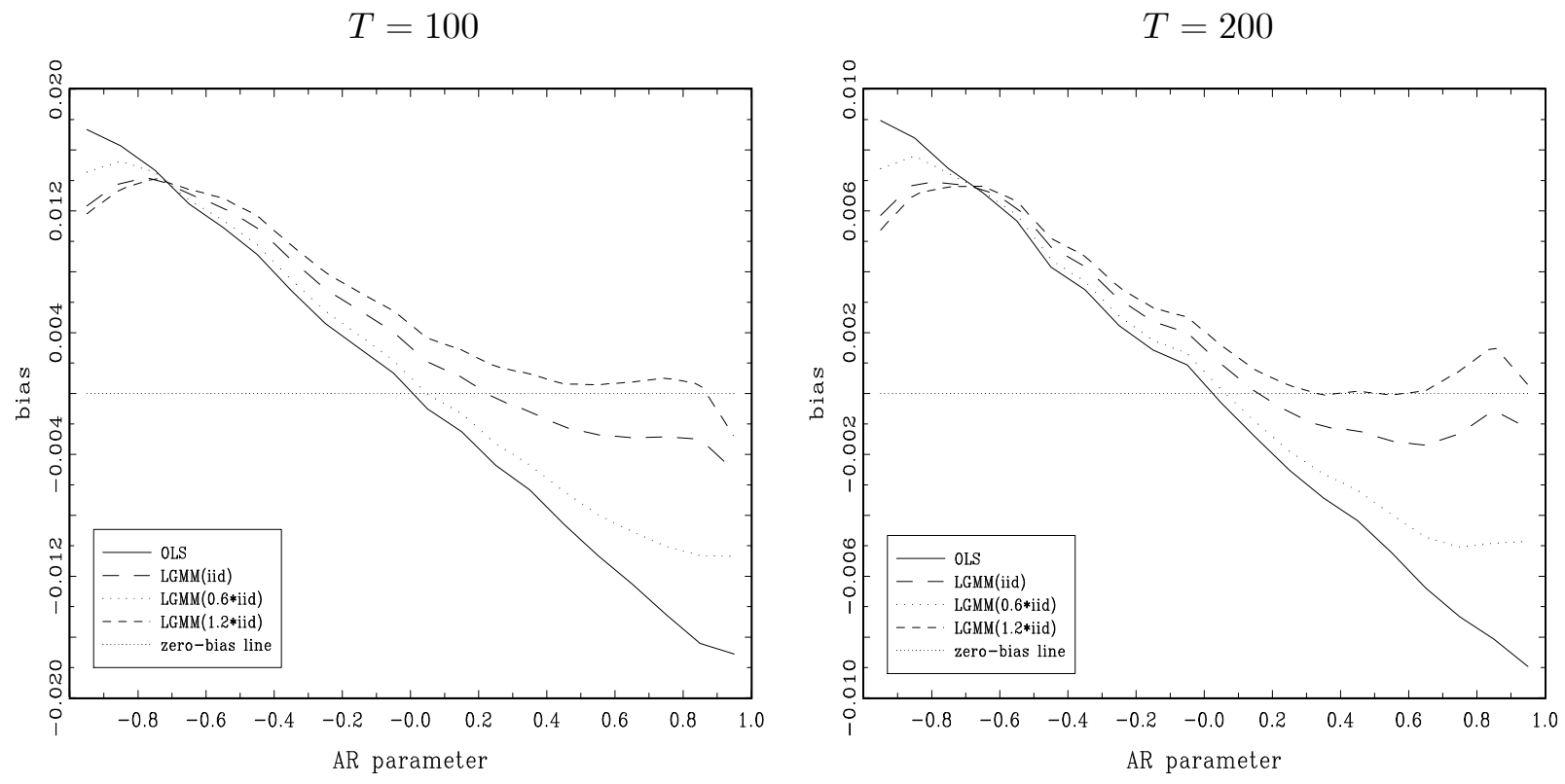

Figure 1. Mean bias of OLS and LGMM estimators in a zero-mean AR(1) model with AR parameter $\theta_{0}=-0.95,-0.85, \ldots, 0.85,0.95$, and $T=100$ (left graph) and $T=200$ (right graph). The LGMM estimators are computed with different bandwidths: (approximate) iid bandwidth $T^{-1 / 5}$, $0.6 T^{-1 / 5}$ and $1.2 T^{-1 / 5}$ with a standardized conditioning variable. The mean bias is computed from 100,000 Monte Carlo replications. 